\title{
Gaussian-consensus filter for nonlinear systems with randomly delayed measurements in sensor networks ${ }^{\text {th }}$
}

\author{
Yanbo Yang, $g^{\mathrm{a}, \mathrm{b}, *}$, Yan Liang, ${ }^{\mathrm{a}, \mathrm{b}}$, Quan Pan ${ }^{\mathrm{a}, \mathrm{b}}$, Yuemei Qin ${ }^{\mathrm{a}, \mathrm{b}}$, Xiaoxu Wang ${ }^{\mathrm{a}, \mathrm{b}}$ \\ ${ }^{a}$ School of Automation, Northwestern Polytechnical University, Xi'an, P.R. China 710072 \\ ${ }^{b}$ Key Laboratory of Information Fusion Technology, Ministry of Education, Xi'an, P.R. \\ China 710072
}

\begin{abstract}
This paper presents the decentralized state estimation problem of discrete-time nonlinear systems with randomly delayed measurements in sensor networks. In this problem, measurement data from the sensor network is sent to a remote processing network via data transmission network, with random measurement delays obeying a Markov chain. Here, we present the Gaussian-consensus filter (GCF) to pursue a tradeoff between estimate accuracy and computing time. It includes a novel Gaussian approximated filter with estimated delay probability (GEDPF) online in the sense of minimizing the estimate error covariance in each local processing unit (PU), and a consensus strategy among PUs in processing network to give a fast decentralized fusion. A numerical example with different measurement delays is simulated to validate the proposed method.

Keywords: Gaussian approximated filter, sensor networks, consensus filter, randomly delayed measurements, Markov chain
\end{abstract}

\section{Introduction}

Considerable research has been undertaken in the field of estimation theory in relation to the discrete-time nonlinear systems over the past several decades due to its widespread applications in process control [1, 2], signal

\footnotetext{
*Corresponding author

Email address: yangyanbo9@mail.nwpu.edu.cn, yangyb8911@gmail.com (Yanbo Yang)
}

Preprint submitted to Information Fusion

October 12, 2015

(C) 2015. This manuscript version is made available under the Elsevier user license http://www.elsevier.com/open-access/userlicense/1.0/ 
${ }_{5}$ processing [3], fault detection and isolation [4], integrated navigation [5] and target tracking [6, 7]. In general, the minimum-mean-square-error estimator for nonlinear systems are almost always intractable in the Bayesian view [8, and hence much attention has been paid on approximation strategies for designing cost-effective estimators. One strategy is the function approximation,

10 i.e., nonlinear dynamic/measurement functions are replaced by piece-wise timevarying linear functions, including the extended Kalman filter [8] via Taylor expansion, the central difference filter [9] based on derivative operation and the divided difference filter [10] based on interpolation polynomial. Generally speaking, function-approximation estimators are computation-effective but sensitive to linearized errors or differential operations. An alternative strategy is the density approximation, i.e., the conditional state probability density function is represented as a Gaussian or Gaussian mixture distribution [11, 12]. This density-approximation strategy results in the integrated framework of analytical computation and numerical integration. Through choosing different numerical integration schemes, the computation burden and estimation accuracy can be balanced. Up to now, the resultant filters are also called Gaussian Approximated Filters (GAFs) including the unscented Kalman filter (UKF) [13] based on unscented transformation, the Gauss-Hermite Filter (GHF) 14] based on Gaussian-Hermite quadrature rule, the new quadrature Kalman filter [15] based 25 on statistical linear regression, the square-root quadrature Kalman filter [16] based on matrix triangularization, the cubature Kalman filter (CKF) [17] based on spherical-radial cubature rule. In general, all these GAFs are limited in the delay-free scope, i.e., the measurement arrives on time.

However, in many actual situations, measurements may arrive at the data processing center with random delays. For example, in a networked multisensor remote-sensing system, sensors may be geographically far away from estimators/controllers and hence the random measurement delay is definitely inevitable due to limited-capability data transmission or additional routing. It motivates the research on state estimation with randomly delayed mea35 surements [18, 19, 20]. A stochastic extended Kalman filter was presented 
for interconnected power systems with partially or totally delayed measurements [21]. The extended and unscented filters were proposed for a class of nonlinear discrete-time stochastic systems with one-step Bernoulli random measurement delay [22]. Moreover, via Gaussian approximations of the one-step posterior predictive probability density functions of state and delayed measurement, a novel GAF was proposed for a nonlinear stochastic systems with onestep randomly Bernoulli delayed measurements [23. Similarly, the corresponding Gaussian smoother was also derived [24. A new unscented filtering was presented for a class of nonlinear stochastic systems with random measurement delays less than three time instants, and this result was extended to deal with multi-step Bernoulli delayed measurements [25]. In general, all these methods have the common assumption that the delay should be Bernoulli distributed with known probability. However, such an assumption may not hold since, in network-based data transmissions, time delays typically occur in a batch mode, and the transition from one mode to another may obey a certain probability distribution [26]. Meanwhile, random delays in a networked system may exhibit the feature that the occurrence of current delay depends on its previous delay [27. Therefore, a seemingly more realistic assumption is that the switching between random delays at adjacent instants abides by a Markov chain, which includes the Bernoulli assumption as a special case [28]. Furthermore, above discussed methods for nonlinear systems with randomly delayed measurements all require centralized processing, i.e., measurements, maybe from different sources, should be collected together and then processed at a fusion center. Actually, the delay occurs inevitably by computation sources being far away from sensors in remote sensing or control. At the same time, many computing units may be deployed, constituting a network in order to accommodate massive sensor data processing. Therefore, it is necessary to develop a decentralized processing and fusion method.

So far, to the best of authors' knowledge, the state estimation and decentralized fusion for nonlinear systems with random measurement delays obeying a Markov chain in sensor networks has not been investigated, which motivates us 
to formulate this new problem. In the considered problem, sensor data collected by a sensor network is sent to a processing network via transmission network. The data transmission incurs the random delay and hence leads to the existence of multi-mode uncertainties and stochastic parameters which are coupled with system nonlinearity and stochastic noises.

The technical contributions of this paper are as follows. Firstly, a Markov process is utilized to depict the random measurement delay in each processing unit $(\mathrm{PU})$. Such modeling is more general and practical, by the fact that the 75 Markov process includes the Bernoulli process which is a widely-accepted random delay model in existing researches [22, 23, 25] as a special case and can exploit the relationship of delays at adjacent sampling instants [26, 27]. Secondly, a novel and generalized GAF with estimated delay probability (GEDPF) is derived for local state estimation in each PU. Here, the delay probability is unknown and even time-varying instead of being known in [22, 23, 25. Meanwhile, the derived GEDPF will be degraded to the common GAF in 14] if there is no delay, or the GAF in [23] if the delay is one-step Bernoulli distributed with known probability. Thirdly, the derived GEDPF is based on lower-dimensional state augmentation, which leads lower-dimensional matrix calculations and Gaussian approximations, compared with the unscented filtering with multi-step random delays in [25]. Fourthly, combined with consensus strategy, the proposed Gaussian-consensus filter (GCF) gives a decentralized fusion implementation with random measurement delays, which has never been investigated for existing filters with delayed measurements in [22, 23, 25] or consensus filters for networked sensors [29, 30, 31].

The rest of this paper is organized as follows. The problem formulation is presented in Section 2. The GEDPF is derived for nonlinear systems with randomly delayed measurements and the GCF is further presented in the processing network in Section 3. A numerical example is simulated in Section 4 to validate the proposed method. Finally, the conclusion is supplied in Section 5. All proofs are presented in the Appendix.

Notation: Throughout this paper, superscripts " -1 " and " $T$ " represent the 
inverse and transpose operation of matrix, respectively. $I$ and $O$ denote the identity and zero matrices of appropriate dimensions, respectively. The symbol ":=" means definition. $E(\cdot)$ and $\operatorname{cov}(\cdot)$ denote mathematical expectation and covariance calculation, respectively. $(\cdot)$ denotes the same content as that in the previous parenthesis. The Gaussian distribution $N\left(\wp_{\rho} ; \hat{\wp}_{\rho \mid k}, P_{\rho \mid k}^{\wp \wp}\right)$ is denoted by $G_{\rho \mid k}(\wp) .\|\cdot\|$ denotes the 2 -norm of a vector.

\section{Problem formulation}

Consider the problem of multi-sensor state estimation for a nonlinear dynamic process as shown in Fig. 1. The whole system contains a sensor network, a transmission network and a processing network. Here, the sensor network contains a large number of clustered sensor nodes to detect and collect state information. Then, the transmission network is in charge of sending sensor measurements to buffers, and measurements are subjected to random delays in transmission. The processing network is constituted by many PUs, and each PU obtains a localized estimate based on the newly-received delayed measurements from its corresponding sensor cluster and exchanges its estimate with its neighbors for estimation consensus.

Here, the random delay may exhibit the feature that the occurrence of current delay depends on its previous delay [27, or the transition from one delayed step to another may obey a certain probability distribution [26]. Thus, a practically random process to describe this delay is the first-order Markov process.

Motivated by this, we formulate a new estimation problem as follows:

$$
\text { nonlinear dynamical process : } x_{k+1}=f_{k}\left(x_{k}\right)+w_{k} \text {, }
$$

the sampled measurement in a sensor node : $z_{k+1}=h_{k+1}\left(x_{k+1}\right)+v_{k+1}$,

where $w_{k}$ and $v_{k}$ are uncorrelated zero-mean Gaussian, white noises satisfying $E\left(w_{k} w_{t}^{T}\right)=Q_{k} \delta_{k t}$ and $E\left(v_{k} v_{t}^{T}\right)=R_{k} \delta_{k t}$, respectively, with $\delta_{k t}$ being the Kro- 


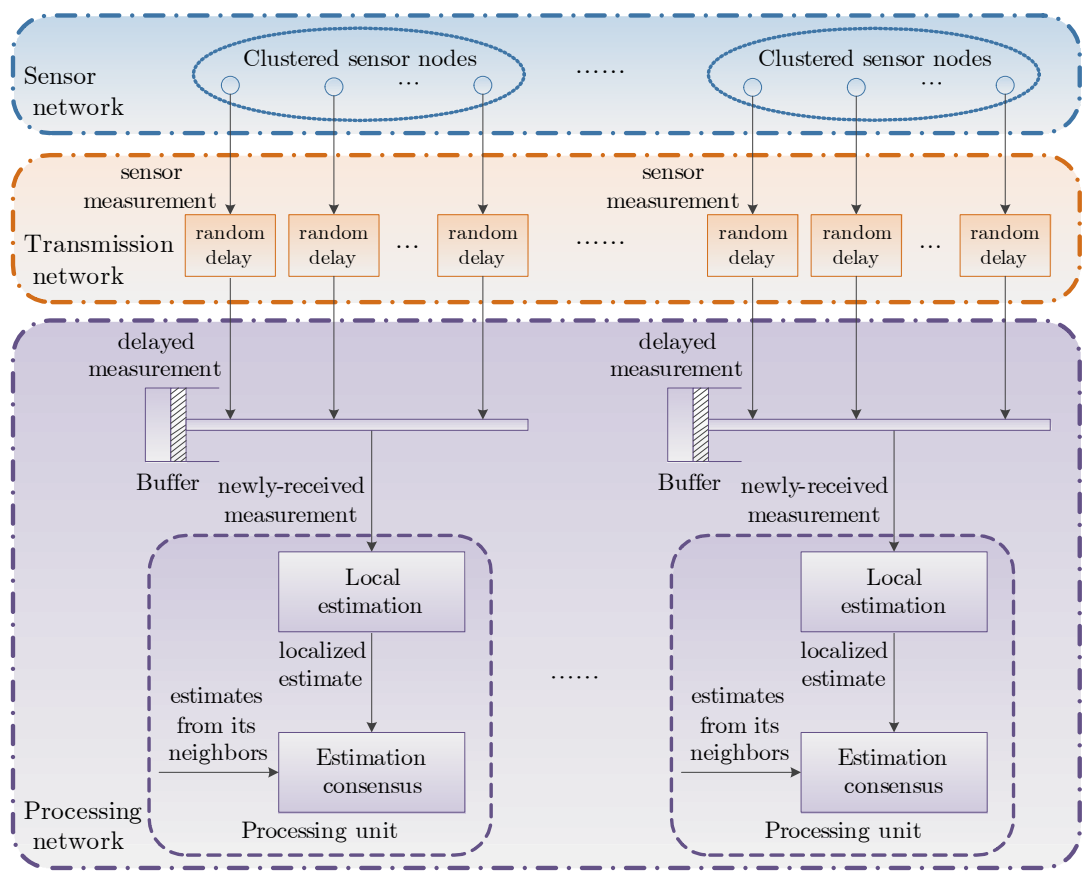

Figure 1: Problem Formulation.

necker delta function. Meanwhile, the initial state is a Gaussian random vector with mean $x_{0}$ and covariance $P_{0}$, being uncorrelated with $w_{k}$ and $v_{k}$.

In an ideal condition, a sensor measurement is transmitted to the corresponding buffer and taken out in time for local state estimation in processing network. However, sensors may be far away from buffers or buffers may be far away from estimators, and the measurement may arrive after the corresponding state estimation in several steps. Thus, random measurement delay due to data transmission and relay or network congestion is definitely inevitable. Consider the case that any an induced latency from a sensor to an estimator in the $m^{\text {th }} \mathrm{PU}$ is not more than $s_{m}$-step sampling period, we will obtain the following measurement output from the corresponding buffer:

the received meaurement in the $m^{t h} \mathrm{PU}: y_{k+1}^{m}=\sum_{j=0}^{\min \left(k+1, s_{m}\right)} \gamma_{j, k+1}^{m} z_{k+1-j}^{m}$,

where $z_{k+1-j}^{m}$ is the sampled measurement from the corresponding sensor node. 
$s_{m}$ denotes the maximum delay step in the $m^{t h} \mathrm{PU} . \gamma_{j, k+1}^{m}$ is a $0-1$ binary random variable obeying a discrete-time Markov chain with the switching probability:

$$
P\left\{\gamma_{j, k+1}^{m}=1 \mid \gamma_{i, k}^{m}=1\right\}=\lambda_{i j, k+1}^{m}, \quad i, j=0,1, \cdots, s_{m}, \quad m=1, \cdots, M
$$

Meanwhile, assume that the newly-received measurement $y_{k+1}$ is only from one sampling instant, and hence the following constraint is given:

$$
\sum_{j=0}^{\min \left(k+1, s_{m}\right)} \gamma_{j, k+1}^{m}=1 .
$$

Moreover, $\gamma_{j, k+1}^{m}$ is uncorrelated with $x_{0}, w_{k}$ and $v_{k}$ in the corresponding sensor node.

Remark 1: It is easily testified that our proposed system (1)-(5) in a PU can be degraded to the nonlinear system without delayed measurements if $P\left\{\gamma_{0, k}^{m}=1\right\}=1$ and $P\left\{\gamma_{j, k}^{m}=1\right\}=0$ for $j=1, \cdots, \min \left(k, s_{m}\right)$. Moreover, the proposed system without measurement delay is also a common nonlinear system in networked sensors. On the other hand, our system in the $m^{\text {th }} \mathrm{PU}$ will be degraded to the nonlinear system with randomly Bernoulli delayed measurements if $\gamma_{i, k}^{m}$ and $\gamma_{j, t}^{m}$ are mutually uncorrelated for any $k \neq t$, due to that the Bernoulli process is only a special case for this Markov process [28]. Furthermore, it is equivalent to the model in [22, 23] with $P\left\{\gamma_{j, k}^{m}=1\right\}=0$ for $j=2, \cdots, \min \left(k, s_{m}\right)$, or to the model in $[25]$ for $j=3, \cdots, \min \left(k, s_{m}\right)$.

Except for the decentralized and distributed structure of processing network, model nonlinearity, stochastic parameters and mode uncertainty caused by random delay coexist in the considered system. Thus, the filter to be designed should own not only the decentralized structure, but also the ability for joint state estimation and parameter identification in the nonlinear case. Based on it, the GCF is proposed in the following section to pursue a tradeoff between estimation accuracy and computation cost. It is expected to obtain not only a novel and generalized GAF for nonlinear systems with randomly delayed measurements in a single PU, but also a scalable and distributed fusion among multiple PUs. 
Remark 2: The GAF is a convenient and widely used filter in nonlinear cases [14, 23]. The Gaussian density belongs to the exponential family and will still be Gaussian after any a linear transformation. This makes the recursive operation being available and the corresponding GAF is analytical, under reasonable Gaussian assumptions. Therefore, in the considered problem, it is rational to design a novel GAF considering random delay in the single PU to obtain an accuracy estimate performance. Meanwhile, due to the existence of random delay, the posterior probability of delay is also identified.

Remark 3: Based on the novel GAF in a single PU, the following three considerations prompt us to give the following decentralized structure. Firstly, the decentralized structure is needed in massive data processing, which can support the multi-core processing or cooperative processing of multiple distributed computing units. Secondly, the computation efficiency is expected to be greatly improved because the consensus strategy avoids computationintensive cross-covariance calculations among local estimate errors in distributed fusion 29, 30, 31, 32. Thirdly, PUs are always geographic proximity and have high-speed data exchange links, which can support information interaction of consensus iteration.

\section{Gaussian-consensus filter for nonlinear systems with randomly de- layed measurements in sensor networks}

In this part, we will firstly derive the GEDPF for nonlinear systems with randomly delayed measurements in a PU, and then the GCF will be presented based on the GEDPF to obtain an asymptotically consistent estimate in the whole processing network.

\subsection{Gaussian approximated filter in a single processing unit}

In this subsection, we will derive the local estimation in the $m^{\text {th }}$ PU. For simplicity, the superscript " $m$ " is omitted without confusion here, for example, $\lambda_{i j, k+1}^{m}$ is simplified as $\lambda_{i j, k+1}$. 
Denote the obtained measurement sequence $\left\{y_{1}, \cdots, y_{l}\right\}$ as $Y_{1: l}$. Define

$$
\hat{\wp}_{k \mid l}:=E\left(\wp_{k} \mid Y_{1: l}\right), \quad P_{k, t \mid l}^{\wp \Im}:=\operatorname{cov}\left(\wp_{k}, \Im_{t} \mid Y_{1: l}\right)=E\left(\tilde{\wp}_{k \mid l} \tilde{\Im}_{t \mid l}\right),
$$

where $\tilde{\wp}_{k \mid l}:=\wp_{k}-\hat{\wp}_{k \mid l}$ and $\tilde{\Im}_{t \mid l}:=\Im_{t}-\hat{\Im}_{t \mid l} . \wp$ and $\Im$ can be used to represent $x, y, z$ and $v$, appropriately. For simplicity, $P_{k, t \mid l}^{\wp \Im}$ is represented as $P_{k \mid l}^{\wp \Im}$ if $k=t$.

Before derivation, some Gaussian assumptions are given to approximate posterior probability densities, which are similar to Gaussian approximations in [14, also being used in other GAFs such as UKF [13] or CKF [17].

Assumption 1: The posterior predictive probability density of the state $x_{k+1}$ conditioned by $Y_{1: k}$ is Gaussian, i.e.,

$$
p\left(x_{k+1} \mid Y_{1: k}\right)=N\left(x_{k+1} ; \hat{x}_{k+1 \mid k}, P_{k+1 \mid k}^{x x}\right) .
$$

Assumption 2: The posterior predictive probability density of the delayed measurement $y_{k+1}$ conditioned by $Y_{1: k}$ is Gaussian, i.e.,

$$
p\left(y_{k+1} \mid Y_{k}\right)=N\left(y_{k+1} ; \hat{y}_{k+1 \mid k}, P_{k+1 \mid k}^{y y}\right) .
$$

Through substituting (2) into (3), we have

$$
y_{k+1}=\sum_{j=0}^{\min (k+1, s)} \gamma_{j, k+1}\left(h_{k+1-j}\left(x_{k+1-j}\right)+v_{k+1-j}\right) .
$$

After inserting (8) into $\hat{y}_{k+1 \mid k}$ and $P_{k+1 \mid k}^{y y}$, it is obvious that recursive formulas of $\hat{y}_{k+1 \mid k}$ and $P_{k+1 \mid k}^{y y}$ are depended on not only the current state prediction $\hat{x}_{k+1 \mid k}$ and estimates $\hat{x}_{k+1-j \mid k}$ at previous instants, but also the sampled measurement noise estimates $\hat{v}_{k+1-j \mid k}$ and related covariances for $j=$ $1, \cdots, \min (k, s)$. Therefore, in deriving a GAF for the system (1)-(5) in a PU, besides $p\left(x_{k+1} \mid Y_{1: k+1}\right)$, we also need the Gaussian approximation $p\left(v_{k+1-j} \mid Y_{1: k+1}\right)$ with its mean $\hat{v}_{k+1-j \mid k+1}$ and covariance $P_{k+1-j \mid k+1}^{v v}$.

Denote the following augmented state vectors: $\vartheta_{k+1, i j}:=\left(x_{k+1-i}^{T}, x_{k+1-j}^{T}\right)^{T}$, $\zeta_{k+1, i j}:=\left(v_{k+1-i}^{T}, v_{k+1-j}^{T}\right)^{T}, \xi_{k+1, i^{\prime} j^{\prime}}:=\left(x_{k+1-i^{\prime}}^{T}, v_{k+1-j^{\prime}}^{T}\right)^{T}$, where $i, j, i^{\prime}, j^{\prime}=$ $0,1, \cdots, \min (k+1, s-1)$ and $i>j$. 
Assumption 3: The posterior predictive probability densities of $\vartheta_{k+1, i j}$, $\zeta_{k+1, i j}$ and $\xi_{k+1, i^{\prime} j^{\prime}}$ conditioned by $Y_{1: k}$ are Gaussian, i.e.,

$$
\begin{aligned}
p\left(\vartheta_{k+1, i j} \mid Y_{1: k}\right) & =N\left(\vartheta_{k+1, i j} ; \hat{\vartheta}_{k+1, i j \mid k}, P_{k+1, i j \mid k}^{\vartheta \vartheta}\right) \\
p\left(\zeta_{k+1, i j} \mid Y_{1: k}\right) & =N\left(\zeta_{k+1, i j} ; \hat{\zeta}_{k+1, i j \mid k}, P_{k+1, i j \mid k}^{\zeta \zeta}\right) \\
p\left(\xi_{k+1, i^{\prime} j^{\prime}} \mid Y_{1: k}\right) & =N\left(\xi_{k+1, i^{\prime} j^{\prime}} ; \hat{\xi}_{k+1, i^{\prime} j^{\prime} \mid k}, P_{k+1, i^{\prime} j^{\prime} \mid k}^{\xi \xi}\right)
\end{aligned}
$$

where $\hat{\wp}_{k+1, m n \mid k}:=E\left(\wp_{k+1, m n} \mid Y_{1: k}\right)$ and $P_{k+1, m n \mid k}^{\wp \wp}:=\operatorname{cov}\left(\wp_{k+1, m n} \mid Y_{1: k}\right)$. can denote $\vartheta, \zeta$ or $\xi$. $m$ and $n$ can represent $i$ or $i^{\prime}$ and $j$ or $j^{\prime}$, respectively.

Corollary 1: Under the Assumption 3, we have

$$
N\left(\wp_{k+1, l r} ; \wp_{k+1, l r \mid k}, P_{k+1, l r \mid k}^{\wp \wp}\right)=N\left(\wp_{k, l-1 r-1} ; \wp_{k, l-1 r-1 \mid k}, P_{k, l-1 r-1 \mid k}^{\wp \wp}\right),
$$
for $l, r=1, \cdots, \min (k+1, s-1)$. Here, $\wp$ can denote $\vartheta, \zeta$ or $\xi$.

Define $\hat{\mu}_{j, k \mid l}:=P\left\{\gamma_{j, k}=1 \mid Y_{1: l}\right\}$ as the estimated delay probability conditioned by $Y_{1: l}$ at $k$ instant for the possible $j^{t h}$-step delay. Theorem 1 presents the following recursive estimation of delay probability.

Theorem 1: Given the estimated delay probability $\hat{\mu}_{i, k \mid k}$ at $k$ instant for $i=0,1, \cdots, \min (k, s)$, the estimated delay probability $\hat{\mu}_{j, k+1 \mid k+1}$ at $k+1$ instant for $j=0,1, \cdots, \min (k+1, s)$ has the following form:

$$
\hat{\mu}_{j, k+1 \mid k+1}=\frac{P\left(y_{k+1} \mid \lambda_{j, k+1}=1, Y_{1: k}\right) \hat{\mu}_{j, k+1 \mid k}}{P\left(y_{k+1} \mid Y_{1: k}\right)},
$$

with

$$
\hat{\mu}_{j, k+1 \mid k}=\sum_{i=0}^{\min (k, s)} \lambda_{i j, k+1} \hat{\mu}_{i, k \mid k} .
$$

Proof: See Appendix A.

In $10, P\left(y_{k+1} \mid \lambda_{j, k+1}=1, Y_{1: k}\right)$ is the probability that $y_{k+1}$ is the sampled sensor measurement $z_{k+1-j}$. Here, $P\left(y_{k+1} \mid \lambda_{j, k+1}=1, Y_{1: k}\right)$ and $P\left(y_{k+1} \mid Y_{1: k}\right)$ in Theorem 1 can be obtained by Gaussian approximations $G_{k+1-j \mid k}(z)$ and $G_{k+1 \mid k}(y)$ with the available measurement $y_{k+1}$ as a sample realization, respectively. Moreover, recursive calculations of means and covariances of these Gaussian approximations are shown in Theorems 2 and 3 below.

Theorem 2: Given $\hat{\xi}_{k, i j \mid k}, P_{k, i j \mid k}^{\xi \xi}, \hat{\zeta}_{k, i j \mid k}, P_{k, i j \mid k}^{\zeta \zeta}(i, j=0,1, \cdots, \min (k, s-$ 1)) and the estimated delay probability $\hat{\mu}_{q, k \mid k}(q=0, \cdots, s)$ at $k$ instant, under Assumptions 1-3, the Gaussian approximation of $p\left(v_{k+1-i} \mid Y_{1: k+1}\right)(i=$ 
$0,1, \ldots, \min (k+1, s-1))$ at $k+1$ instant has the following mean $\hat{v}_{k+1-i \mid k+1}$ and covariance $P_{k+1-i \mid k+1}^{v v}$ :

$$
\begin{gathered}
\hat{v}_{k+1-i \mid k+1}=\hat{v}_{k+1-i \mid k}+K_{k+1-i, k+1}^{v}\left(y_{k+1}-\hat{y}_{k+1 \mid k}\right), \\
P_{k+1-i \mid k+1}^{v v}=P_{k+1-i \mid k}^{v v}-K_{k+1-i, k+1}^{v} P_{k+1 \mid k}^{y y}\left(K_{k+1-i, k+1}^{v}\right)^{T},
\end{gathered}
$$

and the corresponding gain

$$
K_{k+1-i, k+1}^{v}=P_{k+1-i, k+1 \mid k}^{v y}\left(P_{k+1 \mid k}^{y y}\right)^{-1},
$$

with

$$
\begin{aligned}
& \hat{v}_{k+1 \mid k}=\mathbf{0}, \\
& P_{k+1 \mid k}^{v v}=R_{k+1}, \\
& \hat{y}_{k+1 \mid k}=\sum_{j=0}^{\min (k+1, s)} \sum_{q=0}^{s} \lambda_{q j, k+1} \hat{\mu}_{q, k \mid k} \hat{z}_{k+1-j \mid k}, \\
& \begin{aligned}
P_{k+1 \mid k}^{y y}= & \sum_{j=0}^{\min (k+1, s)} \sum_{q=0}^{s} \lambda_{q j, k+1} \hat{\mu}_{q, k \mid k}\left(P_{k+1-j \mid k}^{z z}+\hat{z}_{k+1-j \mid k} \hat{z}_{k+1-j \mid k}^{T}\right), \\
& -\hat{y}_{k+1 \mid k} \hat{y}_{k+1 \mid k}^{T}
\end{aligned} \\
& P_{k+1 \mid k}^{v y}=\sum_{q=0}^{s} \lambda_{q 0, k+1} \hat{\mu}_{q, k \mid k} R_{k+1}, \\
& P_{k+1-r, k+1 \mid k}^{v y} \\
& =\sum_{j=0}^{\min (k+1, s)} \sum_{q=0}^{s} \lambda_{q j, k+1} \hat{\mu}_{q, k \mid k} \int v_{k+1-r} h_{k+1-j}^{T}\left(x_{k+1-j}\right) G_{k+1, j r \mid k}(\xi) d \xi_{k+1, j r} \\
& +\sum_{l=1}^{\min (k+1, s)} \sum_{q=0}^{s} \lambda_{q l, k+1} \hat{\mu}_{q, k \mid k} \int v_{k+1-r} v_{k+1-l}^{T} G_{k+1, r l \mid k}(\zeta) d \zeta_{k+1, r l} \\
& -\hat{v}_{k+1-r \mid k} y_{k+1 \mid k}^{T}
\end{aligned}
$$

where

$$
\hat{z}_{k+1 \mid k}=\int h_{k+1}\left(x_{k+1}\right) G_{k+1 \mid k}(x) d x_{k+1},
$$




$$
\begin{gathered}
\hat{z}_{k+1-r \mid k}=\int\left(h_{k+1-r}\left(x_{k+1-r}\right)+v_{k+1-r}\right) G_{k+1, r r \mid k}(\xi) d \xi_{k+1, r r}, \\
P_{k+1 \mid k}^{z z}=R_{k+1}+\int h_{k+1}\left(x_{k+1}\right) h_{k+1}^{T}\left(x_{k+1}\right) G_{k+1 \mid k}(x) d x_{k+1}-\hat{z}_{k+1 \mid k} \hat{z}_{k+1 \mid k}^{T} \\
P_{k+1-r \mid k}^{z z}=\int\left(h_{k+1-r}\left(x_{k+1-r}\right)+v_{k+1-r}\right)(\cdot)^{T} G_{k+1, r r \mid k}(\xi) d \xi_{k+1, r r} \\
-\hat{z}_{k+1-r \mid k} \hat{z}_{k+1-r \mid k}^{T}
\end{gathered}
$$

for $r=1, \cdots, \min (k+1, s-1)$.

Proof: See Appendix B.

Given the first two moments of the posterior $p\left(v_{k+1-i} \mid Y_{1: k+1}\right)$ from Theorem 2 , we can obtain the final first two moments of original state $x_{k+1}$ as follows.

Theorem 3: Given $\hat{\xi}_{k, i j \mid k}, P_{k, i j \mid k}^{\xi \xi}, \hat{\zeta}_{k, i j \mid k}, P_{k, i j \mid k}^{\zeta \zeta}, \hat{\vartheta}_{k, i j \mid k}, P_{k, i j \mid k}^{\vartheta \vartheta}(i, j=$ $0,1, \cdots, \min (k, s-1))$ and the estimated delay probability $\hat{\mu}_{q, k \mid k}(q=0, \cdots, s)$ at $k$ instant, under Assumptions 1-3, the Gaussian approximation of $p\left(x_{k+1-i} \mid Y_{1: k+1}\right)$ $(i=0,1, \cdots, \min (k+1, s-1))$ has the mean $\hat{x}_{k+1-i \mid k+1}$ and covariance $P_{k+1-i \mid k+1}$ at $k+1$ instant as follows.

$$
\begin{gathered}
\hat{x}_{k+1-i \mid k+1}=\hat{x}_{k+1-i \mid k}+K_{k+1-i, k+1}^{x}\left(y_{k+1}-\hat{y}_{k+1 \mid k}\right), \\
P_{k+1-i \mid k+1}^{x x}=P_{k+1-i \mid k}^{x x}-P_{k+1-i, k+1 \mid k}^{x y}\left(P_{k+1 \mid k}^{y y}\right)^{-1}\left(P_{k+1-i, k+1 \mid k}^{x y}\right)^{T},
\end{gathered}
$$

and the corresponding gain

$$
K_{k+1-i, k+1}^{x}=P_{k+1-i, k+1 \mid k}^{x y}\left(P_{k+1 \mid k}^{y y}\right)^{-1},
$$

with

$$
\begin{gathered}
\hat{x}_{k+1 \mid k}=\int f_{k}\left(x_{k}\right) G_{k \mid k}(x) d x_{k}, \\
P_{k+1 \mid k}^{x x}=\int f_{k}\left(x_{k}\right) f_{k}^{T}\left(x_{k}\right) G_{k \mid k}(x) d x_{k}+Q_{k}-\hat{x}_{k+1 \mid k} \hat{x}_{k+1 \mid k}^{T},
\end{gathered}
$$




$$
P_{k+1-i, k+1 \mid k}^{x y}=\sum_{j=0}^{\min (k+1, s)} \sum_{q=0}^{s} \lambda_{q j, k+1} \hat{\mu}_{q, k \mid k} P_{k+1-i, k+1-j \mid k}^{x z}
$$

where

$$
\begin{aligned}
& P_{k+1 \mid k}^{x z}=\int x_{k+1} h_{k+1}^{T}\left(x_{k+1}\right) G_{k+1 \mid k}(x) d x_{k+1}-\hat{x}_{k+1 \mid k} \hat{z}_{k+1 \mid k}, \\
& P_{k+1, k+1-l \mid k}^{x z}=\int f_{k}\left(x_{k}\right) h^{T}\left(x_{k+1-l}\right) G_{k, 0 l-1 \mid k}(\vartheta) d \vartheta_{k, 0 l-1} \\
& +\int f_{k}\left(x_{k}\right) v_{k+1-l}^{T} G_{k, 0 l-1 \mid k}(\xi) d \xi_{k, 0 l-1}-\hat{x}_{k+1 \mid k} \hat{z}_{k+1-l \mid k}^{T} \\
& P_{k+1-r, k+1 \mid k}^{x z}=\int x_{k+1-r} h_{k+1}^{T}\left(x_{k+1}\right) G_{k+1, r 0 \mid k}(\vartheta) d \vartheta_{k+1, r 0}-\hat{x}_{k+1-r \mid k} \hat{z}_{k+1 \mid k}^{T} \\
& P_{k+1-r, k+1-l \mid k}^{x z}=\int x_{k+1-r} h_{k+1-l}^{T}\left(x_{k+1-l}\right) G_{k+1, r l \mid k}(\vartheta) d \vartheta_{k+1, r l} \\
& +\int x_{k+1-r} v_{k+1-l}^{T} G_{k+1, r l \mid k}(\xi) d \xi_{k+1, r l}-\hat{x}_{k+1-r \mid k} \hat{z}_{k+1-l \mid k}^{T}
\end{aligned}
$$

for $r=1, \cdots, \min (k+1, s-1)$ and $l=1, \cdots, \min (k+1, s)$.

Proof: See Appendix C.

From Theorems 2-3, before obtaining the final state estimate $\hat{x}_{k+1 \mid k+1}$ and $P_{k+1 \mid k+1}$, it needs to derive the first two moments about augmented vectors.

Theorem 4: Given $\hat{\xi}_{k, i j \mid k}, P_{k, i j \mid k}^{\xi \xi}, \hat{\zeta}_{k, i j \mid k}, P_{k, i j \mid k}^{\zeta \zeta}, \hat{\vartheta}_{k, i j \mid k}$ and $P_{k, i j \mid k}^{\vartheta \vartheta}$ for $i, j=0,1, \cdots, \min (k, s-1)$ at $k$ instant, the Gaussian approximations of $p\left(\vartheta_{k+1, i j} \mid Y_{1: t}\right), p\left(\zeta_{k+1, i j} \mid Y_{1: t}\right)$ and $p\left(\xi_{k+1, i j} \mid Y_{1: t}\right)(t=k$ or $k+1)$ for $i, j=$ $0,1, \cdots, \min (k+1, s-1)$ has the following unified form:

$$
\begin{aligned}
& \hat{\vartheta}_{k+1, i j \mid t}=\left(\hat{x}_{k+1-i \mid t}^{T}, \hat{x}_{k+1-j \mid t}^{T}\right)^{T}, \\
& \hat{\zeta}_{k+1, i j \mid t}=\left(v_{k+1-i \mid t}^{T}, v_{k+1-j \mid t}^{T}\right)^{T}, \\
& \hat{\xi}_{k+1, i j \mid t}=\left(\hat{x}_{k+1-i \mid t}^{T}, \hat{v}_{k+1-j \mid t}^{T}\right)^{T},
\end{aligned}
$$




$$
\begin{aligned}
& P_{k+1, i j \mid t}^{\vartheta \vartheta}=\left[\begin{array}{cc}
P_{k+1-i \mid t}^{x x} & P_{k+1-i, k+1-j \mid t}^{x x} \\
\left(P_{k+1-i, k+1-j \mid t}^{x x}\right)^{T} & P_{k+1-j \mid t}^{x x}
\end{array}\right], \\
& P_{k+1, i j \mid t}^{\zeta \zeta}=\left[\begin{array}{cc}
P_{k+1-i \mid t}^{v v} & P_{k+1-i, k+1-j \mid t}^{v v} \\
\left(P_{k+1-i, k+1-j \mid t}^{v v}\right)^{T} & P_{k+1-j \mid t}^{v v}
\end{array}\right], \\
& P_{k+1, i j \mid t}^{\xi \xi}=\left[\begin{array}{cc}
P_{k+1-i \mid t}^{x x} & P_{k+1-i, k+1-j \mid t}^{x v} \\
\left(P_{k+1-i, k+1-j \mid t}^{x v}\right)^{T} & P_{k+1-j \mid t}^{x x}
\end{array}\right],
\end{aligned}
$$

where

$$
\begin{aligned}
& P_{k+1-i, k+1-j \mid k+1}^{x x}=P_{k+1-i, k+1-j \mid k}^{x x}-K_{k+1-i, k+1}^{x} P_{k+1 \mid k}^{y y}\left(K_{k+1-j, k+1}^{x}\right)^{T}, \\
& P_{k+1-i, k+1-j \mid k+1}^{v v}=P_{k+1-i, k+1-j \mid k}^{v v}-K_{k+1-i, k+1}^{v} P_{k+1 \mid k}^{y y}\left(K_{k+1-j, k+1}^{v}\right)^{T}, \\
& P_{k+1-i, k+1-j \mid k+1}^{x v}=P_{k+1-i, k+1-j \mid k}^{x v}-K_{k+1-i, k+1}^{x} P_{k+1 \mid k}^{y y}\left(K_{k+1-j, k+1}^{v}\right)^{T},
\end{aligned}
$$

with

$$
\begin{gathered}
P_{k+1, k+1-r \mid k}^{x x}=\int f_{k}\left(x_{k}\right) x_{k+1-r}^{T} G_{k, 0 r-1 \mid k}(\vartheta) d \vartheta_{k, 0 r-1}-\hat{x}_{k+1 \mid k} \hat{x}_{k+1-r \mid k}^{T}, \\
P_{k+1-l, k+1 \mid k}^{v v}=O, \\
P_{k+1-i, k+1 \mid k}^{x v}=O, \\
P_{k+1, k+1-r \mid k}^{x v}=\int f_{k}\left(x_{k}\right) v_{k+1-r}^{T} G_{k, 0 r-1 \mid k}(\xi) d \xi_{k, 0 r-1}-\hat{x}_{k+1 \mid k} \hat{v}_{k+1-r \mid k}^{T} .
\end{gathered}
$$

for $l, r=1, \cdots, \min (k+1, s-1)$.

Proof: See Appendix D.

For the proposed GEDPF, it needs to execute from the one-step randomly delayed case to $s$-step randomly delayed case step-by-step until $k \geq s$. 


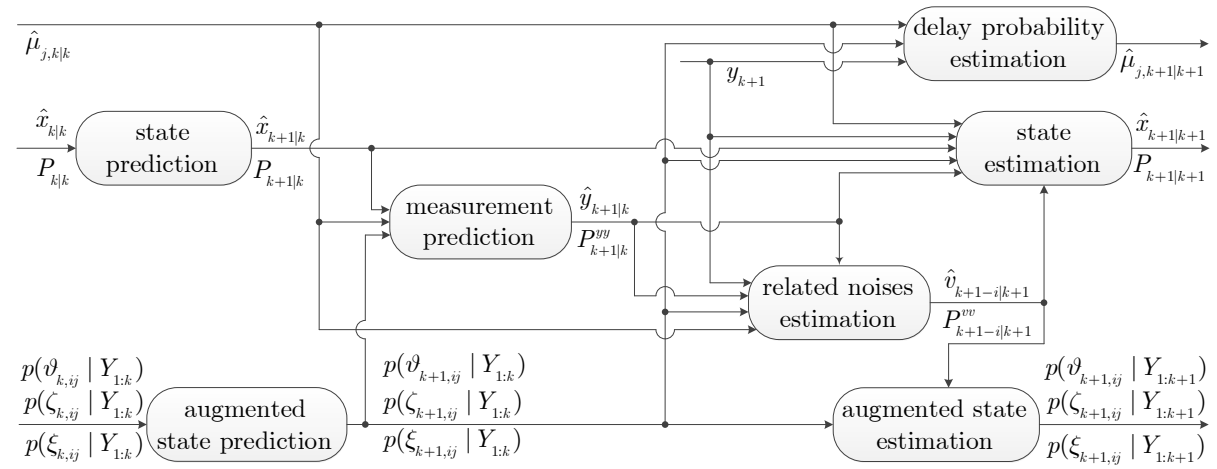

Figure 2: Recursive structure of the proposed GEDPF.

The recursive structure of the proposed GEDPF is shown in Fig. 2. Due to the unknown random delay, the "delay probability estimation" block is needed, which corresponds to Theorem 1. In recursive state estimation, the newlyreceived measurement prediction is required, which leads to the "measurement prediction" block. Moreover, due to the random delay in arriving measurement, there is a need for sampled measurement noises estimation, which produces the "related noises estimation" block. These two blocks correspond to Theorem 2. Then, based on outputs of "state prediction" and "state estimation" blocks corresponding to Theorem 3, the final state estimate can be obtained. In addition, "augmented state prediction" and "augmented state estimation" blocks provide additionally intermediate estimates, which correspond to Theorem 4.

Remark 4: If the variable $\gamma_{j, k}$ in the proposed system (1)-(3) is Bernoulli distributed, the proposed GEDPF will be degraded for nonlinear systems with randomly Bernoulli distributed delayed measurements. At this moment, the delay probability $\hat{\mu}_{j, k+1 \mid k+1}$ can be estimated as follows. The first is to calculate the probability $P\left(y_{k+1} \mid \gamma_{j, k+1}=1, Y_{1: k}\right)$ for each possible delay with the newlyreceived measurement $y_{k+1}$ and normalize it. Then, it can be regarded as the delay probability at current instant, due to that measurement delays are not correlated at adjacent instants. Furthermore, if there is a priori knowledge of the delay probability $\mu_{j, k+1}$, it can be used to replace the predicted delay 


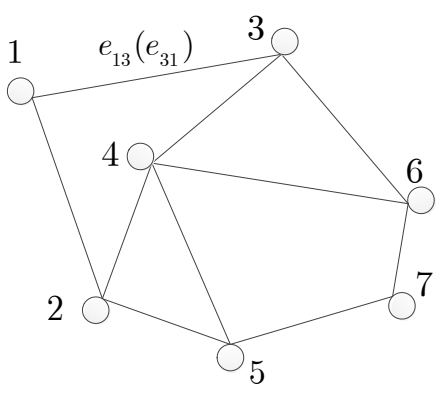

Figure 3: The processing network denoted as an undirected Graph.

probability $\sum_{q=0}^{s} \lambda_{q j, k+1} \hat{\mu}_{q, k \mid k}$ in Theorems 2 and 3 directly.

Remark 5: It is worth mentioning that the derived GEDPF is different from those in [14, 22, 23, 25. Firstly, it owns a general framework due to that it will be degraded to the GAF in [23] if the delay is one-step randomly Bernoulli distributed with known probability, or a common structure of GAF as that in [13, 14, 15, 16, 17] if there is no delay. Secondly, the considering measurement delay is more general and practical than those in [22, 23, 25] due to the Bernoulli process being a special case of Markov process. Thirdly, compared with the unscented filtering with direct state augmentation based on multistep random delay in 25, the derived GEDPF is based on lower-dimensional augmented states, leading to a lower-dimensional Gaussian approximation and matrix calculation.

\subsection{Gaussian-consensus filter in the processing network}

After obtaining the GEDPF in a PU, we will present a decentralized method to fuse all localized estimates. The processing network is denoted as an undirected graph $G=(\mathcal{V}, \mathcal{E})$, with an example shown in Fig. 3. A vertex $m \in \mathcal{V}=$ $\{1, \cdots, M\}$ represents a PU and an edge $e_{m n} \in \mathcal{E}=\left\{e_{m n}, m, n=1, \cdots, M\right\}$ means the permission about information sharing between these two neighbors. Let $\mathcal{N}_{m}=\left\{m \in \mathcal{V}: e_{m n} \in \mathcal{E}\right\}$ denote the set of neighbors of the $m^{\text {th }} \mathrm{PU}$, and $\mathcal{J}_{m}=\mathcal{N}_{m} \cup\{m\}$. Based on the consensus algorithms in 31, the following formulation is presented to obtain a final consistent estimate in processing 


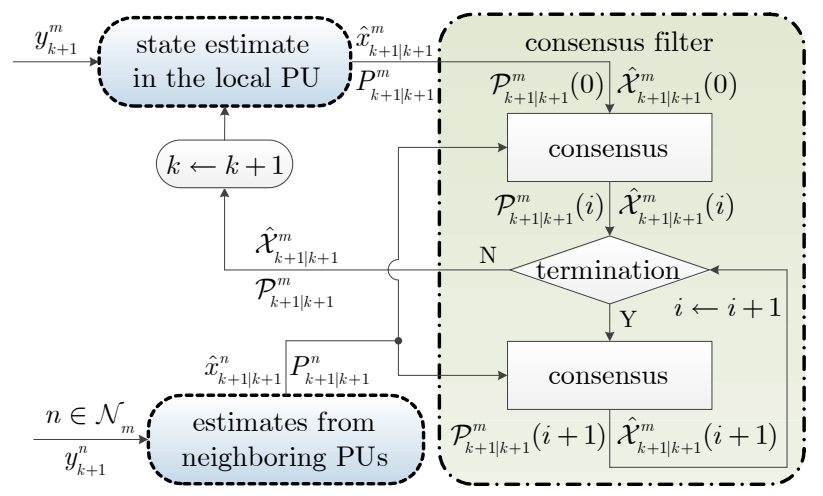

Figure 4: Recursive structure of the proposed GCF.

network, with its recursive structure shown in Fig. 4.

$$
\begin{aligned}
\hat{\mathcal{X}}_{k+1 \mid k+1}^{m}(i+1)= & \hat{\mathcal{X}}_{k+1 \mid k+1}^{m}(i)+\varepsilon \sum_{n \in \mathcal{N}_{m}}\left(\hat{\mathcal{X}}_{k+1 \mid k+1}^{n}(i)-\hat{\mathcal{X}}_{k+1 \mid k+1}^{m}(i)\right) \\
& +\varepsilon \sum_{n \in \mathcal{J}_{m}}\left(\hat{x}_{k+1 \mid k+1}^{n}-\hat{\mathcal{X}}_{k+1 \mid k+1}^{m}(i)\right) \\
\mathcal{P}_{k+1 \mid k+1}^{m}(i+1)= & \mathcal{P}_{k+1 \mid k+1}^{m}(i)+\varepsilon \sum_{n \in \mathcal{N}_{m}}\left(\mathcal{P}_{k+1 \mid k+1}^{n}(i)-\mathcal{P}_{k+1 \mid k+1}^{m}(i)\right) \\
& +\varepsilon \sum_{n \in \mathcal{J}_{m}}\left(P_{k+1 \mid k+1}^{n}-\mathcal{P}_{k+1 \mid k+1}^{m}(i)\right)
\end{aligned}
$$

where $\hat{\mathcal{X}}_{k+1 \mid k+1}^{m}(i)$ and $\mathcal{P}_{k+1 \mid k+1}^{m}(i)$ are estimates in the $m^{\text {th }} \mathrm{PU}$ at $k+1$ instant in the $i^{\text {th }}$ iteration step and $\varepsilon$ is the iteration step size. Initial values $\hat{\mathcal{X}}_{k+1 \mid k+1}^{m}(0)$ and $\mathcal{P}_{k+1 \mid k+1}^{m}(0)$ are corresponding estimates in 25) and 26) from the GEDPF in the $m^{\text {th }}$ PU. $\hat{x}_{k+1 \mid k+1}^{j}$ and $P_{k+1 \mid k+1}^{j}$ are treated as inputs, which are the estimated state and covariance in (25) and (26) from the GEDPF in the $j^{\text {th }} \mathrm{PU}$, as shown in the block "estimates from neighboring PUs" in Fig. 4. The block "consensus filter" in Fig. 4 shows iteration calculations within 48) and 49). The termination condition of the iteration is $\left\|\mathcal{X}_{k+1 \mid k+1}^{m}(i)-\mathcal{X}_{k+1 \mid k+1}^{m}(i-1)\right\| \leq$ $\hbar$ or $i \leq \ell_{\max }$, where $\hbar$ is a threshold value tending to zero and $\ell_{\max }$ is the maximum iteration step.

The key point of implementing the GCF is to compute Gaussian integrals. A common way is to adopt the numerical integral method as similar as those in 
Step 1. Initialization.

Set initial values $\hat{x}_{0 \mid 0}, P_{0 \mid 0}^{x x}$, and the delay probability $\hat{\mu}_{j, 0 \mid 0}$ in each PU.

Step 2. Localized state estimate in a PU.

a. Calculate $\hat{x}_{k+1 \mid k}$ and $P_{k+1 \mid k}^{x x}$ by 28 and 29 in Theorem 3 , respectively.

b. Let $t=k$ and obtain means and covariances of $p\left(\vartheta_{k+1, i j} \mid Y_{1: t}\right), p\left(\zeta_{k+1, i j} \mid Y_{1: t}\right)$ and $p\left(\xi_{k+1, i j} \mid Y_{1: t}\right)$ by $35-40$ in Theorem 4.

c. Calculate $\hat{y}_{k+1 \mid k}$ and $P_{k+1 \mid k}^{y y}$ by 17 and 18 in Theorem 2 , respectively.

d. Obtain $\hat{v}_{k+1-i \mid k+1}$ and $P_{k+1-i \mid k+1}^{v v}$ by 12 and 13 in Theorem 2, respectively, where $K_{k+1-i \mid k+1}^{v}$ is calculated by 14 for $i=0,1, \cdots, \min (k+1, s-1)$.

e. Obtain $\hat{x}_{k+1-i \mid k+1}$ and $P_{k+1-i \mid k+1}^{x x}$ by 25 and 26 in Theorem 3 , respectively, where $K_{k+1-i \mid k+1}^{x}$ is calculated by (27) for $i=0,1, \cdots, \min (k+1, s-1)$.

f. Calculate means and covariances of $p\left(\vartheta_{k+1, i j} \mid Y_{1: k+1}\right), p\left(\zeta_{k+1, i j} \mid Y_{1: k+1}\right)$ and $p\left(\xi_{k+1, i j} \mid Y_{1: k+1}\right)$ using 35 - 40 for $i, j=0,1, \cdots, \min (k+1, s-1)$.

g. Calculate the estimated delay probability $\hat{\mu}_{j, k+1 \mid k+1}$ by 10 in Theorem 1 for $j=0,1, \cdots, \min (k+1, s-1)$.

Step 3. Consensus filter.

Set the local state estimates in Step 2 as $\hat{\mathcal{X}}_{k+1 \mid k+1}^{m}(0)$ and $\mathcal{P}_{k+1 \mid k+1}^{m}(0)$ for $i=0$. By using 48 and 49 , calculate $\hat{\mathcal{X}}_{k+1 \mid k+1}^{m}(i+1)$ and $\mathcal{P}_{k+1 \mid k+1}^{m}(i+1)$ iteratively until satisfying the termination condition.

Step 4. Recursion.

Set $k \leftarrow k+1$, initialize estimates $\hat{x}_{k \mid k}$ and $P_{k \mid k}^{x x}$ in each $\mathrm{PU}$ as the ultimate iterative values and go back to Step 2.

UKF [13, 33], GHF [14], CKF [17], and so on. A brief computational procedure for the proposed GCF is listed in Table 1 . 
Table 2: The time-varying probability $P\left(\rho_{k}^{m}=1\right)$ in Case 1

\begin{tabular}{ccccc}
\hline time interval & {$[1,50]$} & {$[51,100]$} & {$[101,150]$} & {$[151200]$} \\
\hline PU 1 & 0.8 & 0.4 & 0.7 & 0.6 \\
PU 2 & 0.6 & 0.5 & 0.5 & 0.8 \\
PU 3 & 0.5 & 0.6 & 0.3 & 0.7 \\
PU 4 & 0.7 & 0.4 & 0.6 & 0.5 \\
PU 5 & 0.5 & 0.3 & 0.1 & 0.3 \\
PU 6 & 0.3 & 0.2 & 0.7 & 0.2 \\
PU 7 & 0.8 & 0.5 & 0.9 & 0.5 \\
\hline
\end{tabular}

\section{Numerical example}

In this section, the following univariate non-stationary growth model is used to validate the proposed GEDPF in a single PU and GCF in a processing network as shown in Fig. 3 .

$$
\left\{\begin{array}{c}
x_{k+1}=0.5 x_{k}+25 \frac{x_{k}}{1+x_{k}^{2}}+8 \cos (1.2 k)+w_{k} \\
z_{k}=\frac{x_{k}^{2}}{5}+v_{k}
\end{array},\right.
$$

where $x_{0}=0, P_{0}=5, Q_{k}=10$ and $R_{k}=1$. The dynamic is run for 200 instants. Here, each sensor node corresponds to a PU. Moreover, the sampled measurement noise in each PU is independent. Two random delay cases are considered with abruptly time-varying delay probabilities:

- Case 1: one-step random delay case with the Bernoulli variable $\rho_{k}^{m}$, i.e., $\gamma_{0, k}^{m}=1-\rho_{k}^{m}$ and $\gamma_{1, k}^{m}=\rho_{k}^{m}$;

- Case 2: two-step random delay case with two Bernoulli variables $\rho_{1, k}^{m}$ and $\rho_{2, k}^{m}$, i.e., $\gamma_{0, k}^{m}=1-\rho_{1, k}^{m}, \gamma_{1, k}^{m}=\rho_{1, k}^{m}\left(1-\rho_{2, k}^{m}\right)$ and $\gamma_{1, k}^{m}=\rho_{1, k}^{m} \cdot \rho_{2, k}^{m}$.

The true delay probabilities in each PU are shown in Tables 2 and 3 in detail.

The GEDPF is compared with the common UKF without considering measurement delay 13. (NDUKF) in both Cases 1 and 2, the unscented Kalman filter for nonlinear systems with one-step delayed measurements [22] (OSUKF) 


Table 3: The time-varying probability $P\left(\rho_{1, k}^{m}=1\right), P\left(\rho_{2, k}^{m}=1\right)$ in C
\begin{tabular}{cccccc} 
time interval & {$[1,25]$} & {$[26,50]$} & {$[51,75]$} & {$[76100]$} \\
\hline PU 1 & $0.8,0.4$ & $0.4,0.6$ & $0.6,0.3$ & $0.3,0.7$ \\
PU 2 & $0.4,0.6$ & $0.7,0.7$ & $0.5,0.5$ & $0.6,0.4$ \\
PU 3 & $0.3,0.5$ & $0.6,0.4$ & $0.8,0.6$ & $0.3,0.8$ \\
PU 4 & $0.7,0.4$ & $0.4,0.3$ & $0.7,0.7$ & $0.6,0.6$ \\
PU 5 & $0.6,0.2$ & $0.2,0.5$ & $0.1,0.2$ & $0.3,0.7$ \\
PU 6 & $0.2,0.5$ & $0.5,0.2$ & $0.6,0.6$ & $0.2,0.3$ \\
PU 7 & $0.8,0.7$ & $0.6,0.6$ & $0.9,0.8$ & $0.7,0.6$ \\
\hline
\end{tabular}

in Case 1 and the unscented Kalman filter for nonlinear systems with two-step randomly delayed measurements [25] (TSUKF) in Case 2, to show its estimate performance in a single PU. Meanwhile, the proposed GCF is compared with the centralized OSUKF (COSUKF) in Case 1 and centralized TSUKF (CTSUKF) in Case 2, where both obtain the corresponding local estimate in each PU and fuse all local estimates with an optimal covariance intersection assuming all estimates are independent.

The initial estimates of the compared methods are identical: $\hat{x}_{0 \mid 0}=0$ and $P_{0 \mid 0}=5$. The delay probabilities in OSUKF, TSUKF, COSUKF and CTSUKF are known and the same with true values in Tables 2 and 3 . In the derived GEDPF, the initial delay probabilities are $\hat{\mu}_{0,0 \mid 0}=\hat{\mu}_{1,0 \mid 0}=0.5$ with the transition probability matrix $\lambda_{11, k}=\lambda_{22, k}=0.95$ for $k=1,2, \cdots$ in one-step random delay case, and $\lambda_{00, k}=\lambda_{11, k}=\lambda_{22, k}=0.96, \lambda_{i^{\prime} j^{\prime}, k}=0.02$ for remained elements in two-step random delay case. In the GCF, $\varepsilon=0.1, \hbar=10^{-6}$ and $\ell_{\max }=50$.

The root mean square errors (RMSEs) of the compared methods in the $7^{\text {th }}$ PU are shown in Figs. 5 and 7, while RMSE ratios between two compared 290 methods are shown in Figs. 6 and 8 with the RMSE of proposed GCF being taken as base, via 10000 Monte Carlo realizations. In Figs. 6 and 8, RMSEs of GEDPF are also from the single $7^{t h}$ PU for both two cases. Average estimation 

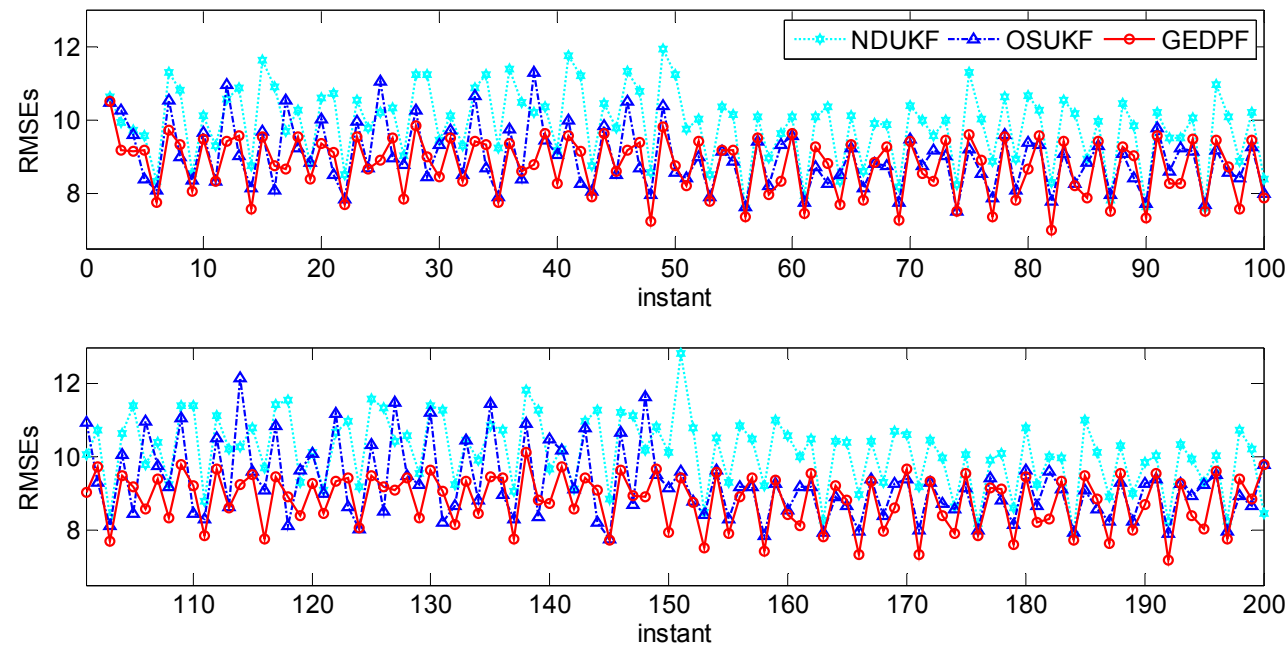

Figure 5: RMSEs of compared methods in the $7^{\text {th }} \mathrm{PU}$ in Case 1.
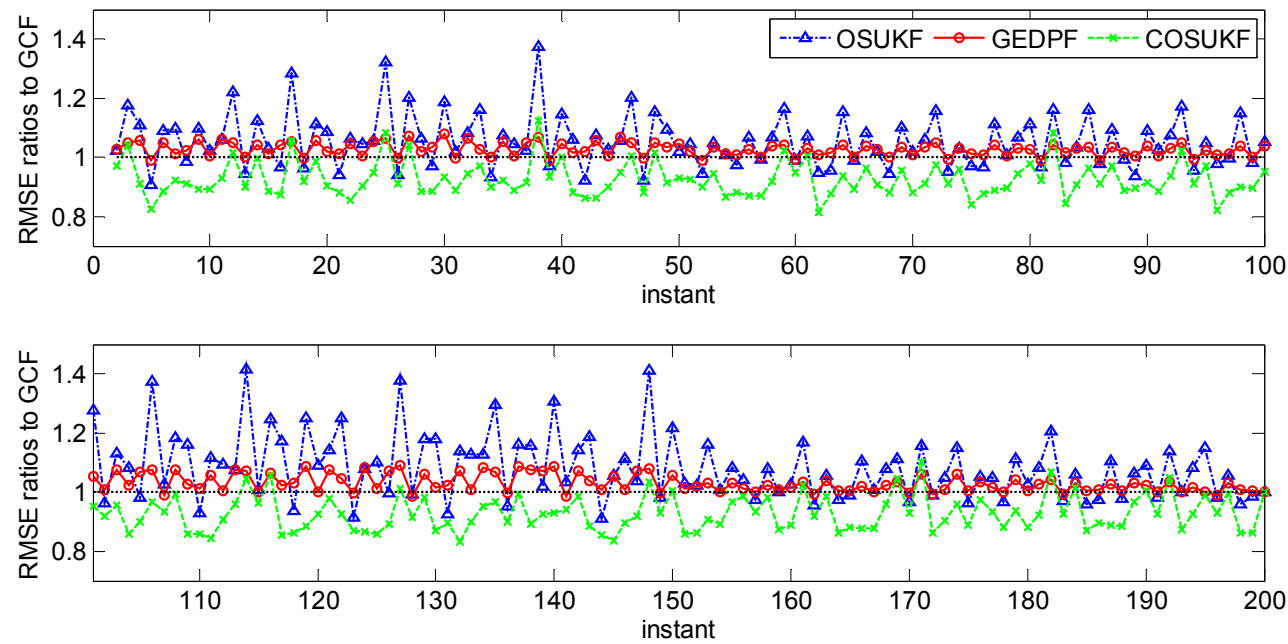

Figure 6: RMSE Ratios to GCF of compared methods in Case 1. 

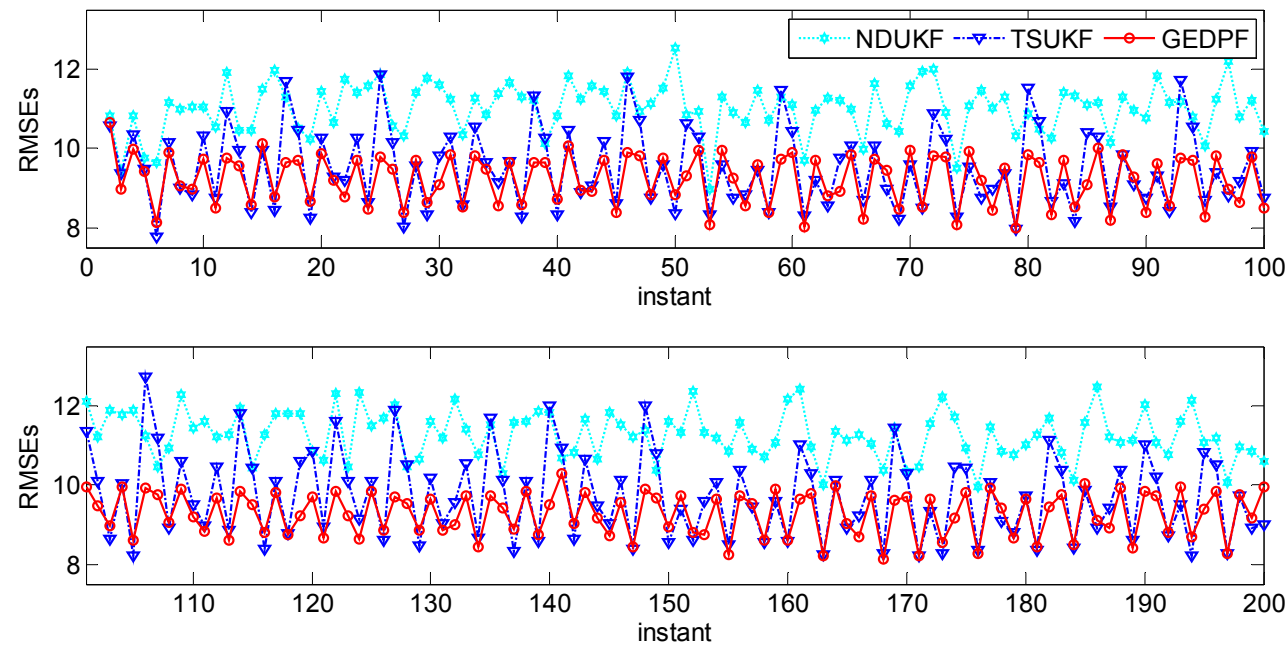

Figure 7: RMSEs of compared methods in the $7^{\text {th }} \mathrm{PU}$ in Case 2.
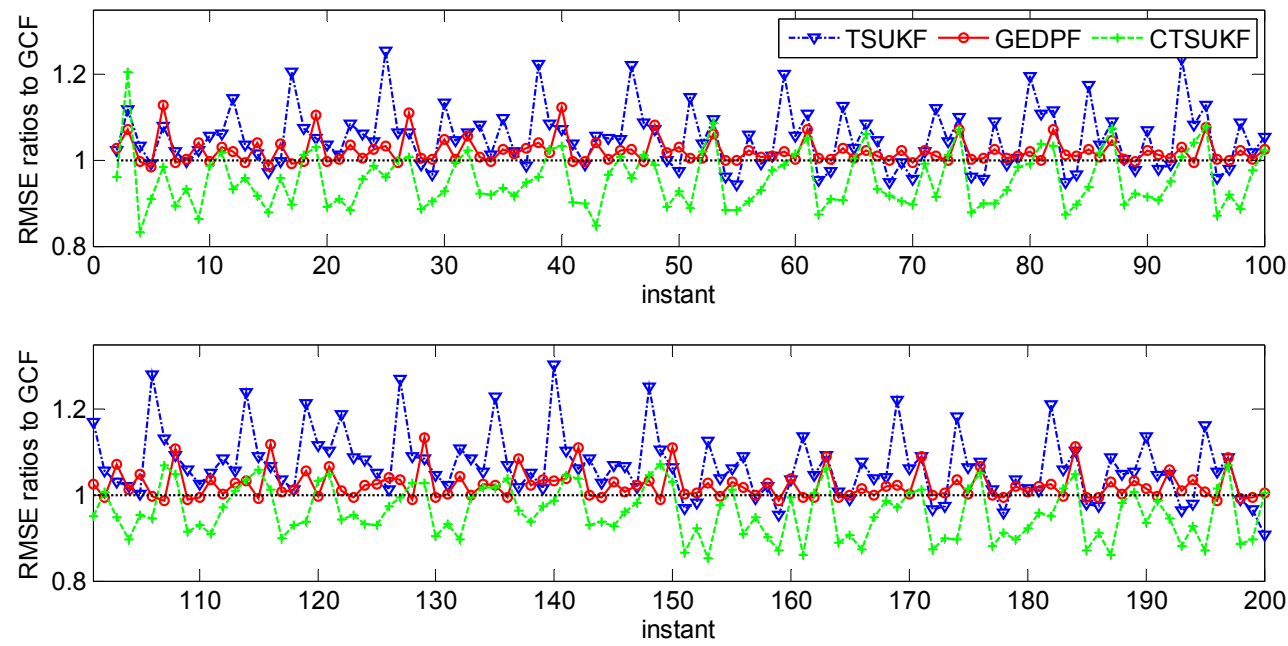

Figure 8: Ratios of RMSEs of compared methods in Case 2. 
Table 4: The average estimation RMSE

\begin{tabular}{cccccccc}
\hline mean & NDUKF & OSUKF & TSUKF & GEDPF & COSUKF & CTSUKC & GCF \\
\hline Case 1 & 9.9960 & 9.1062 & - & $\mathbf{8 . 7 8 8 1}$ & 7.9160 & - & $\mathbf{8 . 5 6 1 0}$ \\
Case 2 & 11.1431 & - & 9.6044 & $\mathbf{9 . 2 4 5 6}$ & - & 8.6646 & $\mathbf{9 . 0 6 9 1}$ \\
\hline \multicolumn{7}{c}{ Table 5: The average computing time (seconds) in a Monte Carlo realization } \\
\hline time & NDUKF & OSUKF & TSUKF & GEDPF & COSUKF & CTSUKC & GCF \\
\hline Case 1 & 0.0195 & 0.0613 & - & $\mathbf{0 . 0 9 6 5}$ & 0.4272 & - & $\mathbf{0 . 0 9 8 2}$ \\
Case 2 & 0.0190 & - & 0.1661 & $\mathbf{0 . 3 0 4 7}$ & - & 1.2412 & $\mathbf{0 . 3 1 7 7}$ \\
\hline
\end{tabular}

RMSEs are listed in Table 4 and the corresponding average computing time in a single Monte Carlo realization is listed in Table 5 by using a computer with Intel i5-4200M CPU @ 2.50GHz.

From Figs. 5 and 7 it can be seen that the RMSE of GEDPF is more stable than others, although all these are small when the delay probability is low or big when the delay probability is high, especially in Case 1. Moreover, the proposed GEDPF has a better estimate accuracy than that of OSUKF and TSUKF in the corresponding case. The main reason is that the delay is estimated in our method online while they just use a vague statistical mean to represen$\mathrm{t}$ it in the OSUKF and TSUKF. Furthermore, TSUKF is derived based on a higher-dimensional Gaussian approximation, where the error of this approximation always becomes bigger with higher dimension. If the delay probability is unknown in OSUKF or TSUKF, its performance will be worse, which can be implied by the NDUKF. In addition, from Figs. 6 and 8 and Table. 4 although the proposed GCF is not as accurate as that of the COSUKF or CTSUKF under the ideal condition, its performance is also approving. Meanwhile, the performance of GCF outperforms than that of the GEDPF, due to more available information improving the final estimate. 
As shown in the Table 5. the average computing time of the GEDPF is low and acceptable in practice, although it is higher than that of OSUKF or TSUKF in the corresponding case. The delay probability estimation in the GEDPF will spend some extra computation cost. Meanwhile, more addition and subtraction operations are included in the GEDPF to estimate intermediate vectors, while it is simple in the TSUKF in 25] due to all estimated vectors being augmented as a higher-dimensional state. In addition, the average computing time of GCF from a single PU is almost the same as that of GEDPF, which is much lower than that of the COSUKF or CTSUKF. This shows the advantage of distributed implementation in saving computing time.

\section{Conclusion}

Considering the state estimation of nonlinear systems in a large-scale system including sensor network, transmission network and processing network, the GEDPF is derived with randomly delayed measurements in a single PU and 325 the GCF is presented for decentralized implementation. The proposed method is a tradeoff between estimate accuracy and computing time. It gives not only a novel and generalized GAF in a single PU with the delay probability estimation online and a low-dimensional Gaussian approximation to obtain a high accurate performance, but also a scalable and distributed algorithm among multiple PUs to gain a fast and effective fusion. A numerical example with different measurement delays validates the proposed GEDPF and GCF.

\section{Acknowledgements}

This work has been supported by China National Science Foundation (No. 61135001, 61374023, 61374519) and the Doctorate Foundation of Northwestern 335 Polytechnical University (No. cx201425). 


\section{Appendix A. Proof of Theorem 1}

According to the Bayesian rule, we have

$$
\hat{\mu}_{j, k+1 \mid k+1}=\frac{P\left(\gamma_{j, k+1}=1, y_{k+1} \mid Y_{1: k}\right)}{P\left(y_{k+1} \mid Y_{1: k}\right)}=\frac{P\left(y_{k+1} \mid \gamma_{j, k+1}=1, Y_{1: k}\right) \hat{\mu}_{j, k+1 \mid k}}{P\left(y_{k+1} \mid Y_{1: k}\right)} .
$$

Furthermore, by the fact that the delay is the first-order Markov chain and $\lambda_{j, k+1}$ is only correlated with $\lambda_{i, k}$ for $i=0,1, \cdots, \min (k, s)$, we have

$$
\begin{aligned}
\hat{\mu}_{j, k+1 \mid k} & =\sum_{i=1}^{\min (k, s)} P\left(\lambda_{j, k+1}=1, \lambda_{i, k}=1 \mid Y_{1: k}\right) \\
& =\sum_{i=1}^{\min (k, s)} P\left(\lambda_{j, k+1}=1 \mid \lambda_{i, k}=1\right) P\left(\lambda_{i, k}=1 \mid Y_{1: k}\right)=\sum_{i=1}^{\min (k, s)} \lambda_{i j, k+1} \hat{\mu}_{i, k \mid k}
\end{aligned} .
$$

\section{Appendix B. Proof of Theorem 2}

Due to $\gamma_{j, k+1}$ being uncorrelated with sampled measurements, we have

$\hat{y}_{k+1 \mid k}=\sum_{j=0}^{\min (k+1, s)} E\left(\gamma_{j, k+1} \mid Y_{1: k}\right) \hat{z}_{k+1-j \mid k}=\sum_{j=0}^{\min (k+1, s)} \sum_{q=0}^{s} \lambda_{q j, k+1} \hat{\mu}_{q, k \mid k} \hat{z}_{k+1-j \mid k}$

where

$$
\begin{gathered}
\hat{z}_{k+1 \mid k}=E\left(h_{k+1}\left(x_{k+1}\right)+v_{k+1} \mid Y_{1: k}\right)=\int h_{k+1}\left(x_{k+1}\right) G_{k+1 \mid k}(x) d x_{k+1}, \\
\hat{z}_{k+1-r \mid k}=\int\left(h_{k+1-r}\left(x_{k+1-r}\right)+v_{k+1-r}\right) G_{k+1, r r \mid k}(\xi) d \xi_{k+1, r r},
\end{gathered}
$$

for $r=1, \cdots, \min (k+1, s)$. Here, $E\left(v_{k+1} \mid Y_{1: k}\right)=\mathbf{0}$.

Based on $E\left(\gamma_{j, k+1}^{2} \mid Y_{1: k}\right)=P\left(\gamma_{j, k+1}=1 \mid Y_{1: k}\right) \times 1=E\left(\gamma_{j, k+1} \mid Y_{1: k}\right)$, we have

$$
\begin{aligned}
& P_{k+1 \mid k}^{y y}=E\left(y_{k+1} y_{k+1}^{T} \mid Y_{1: k}\right)-\hat{y}_{k+1 \mid k} \hat{y}_{k+1 \mid k}^{T} \\
= & E\left(\sum_{j=0}^{\min (k+1, s)} \gamma_{j, k+1} z_{k+1-j} \sum_{j=0}^{\min (k+1, s)} \gamma_{j, k+1} z_{k+1-j} \mid Y_{1: k}\right)-\hat{y}_{k+1 \mid k} \hat{y}_{k+1 \mid k}^{T} \\
= & \sum_{j=0}^{\min (k+1, s)} E\left(\gamma_{j, k+1}^{2} \mid Y_{1: k}\right) E\left(z_{k+1-j} z_{k+1-j}^{T} \mid Y_{1: k}\right)-\hat{y}_{k+1 \mid k} \hat{y}_{k+1 \mid k}^{T} \\
= & \sum_{j=0}^{\min (k+1, s)} \sum_{q=0}^{s} \lambda_{q j, k+1} \hat{\mu}_{q, k \mid k}\left(P_{k+1-j \mid k}^{z z}+\hat{z}_{k+1-j \mid k} \hat{z}_{k+1-j \mid k}^{T}\right)-\hat{y}_{k+1 \mid k} \hat{y}_{k+1 \mid k}^{T}
\end{aligned}
$$


where

$$
\begin{aligned}
& P_{k+1 \mid k}^{z z}=E\left(h_{k+1}\left(x_{k+1}\right) h_{k+1}^{T}\left(x_{k+1}\right) \mid Y_{1: k}\right)+R_{k+1}-\hat{z}_{k+1 \mid k} \hat{z}_{k+1 \mid k}^{T} \\
& =\int h_{k+1}\left(x_{k+1}\right) h_{k+1}^{T}\left(x_{k+1}\right) G_{k+1 \mid k}(x) d x_{k+1}+R_{k+1}-\hat{z}_{k+1 \mid k} \hat{z}_{k+1 \mid k}^{T} \\
& P_{k+1-r \mid k}^{z z}=E\left(z_{k+1-r} z_{k+1-r}^{T} \mid Y_{1: k}\right)-\hat{z}_{k+1-r \mid k} \hat{z}_{k+1-r \mid k}^{T} \\
& =\int\left(h_{k+1-r}\left(x_{k+1-r}\right)+v_{k+1-r}\right)(\cdot)^{T} G_{k+1, r r \mid k}\left(\xi_{k+1, r r}\right) d \xi_{k+1, r r} . \\
& -\hat{z}_{k+1-r \mid k} \hat{z}_{k+1-r \mid k}^{T}
\end{aligned}
$$

By the fact that $v_{k+1}$ is uncorrelated with $Y_{1: k}$, it is obvious that

$$
p\left(v_{k+1} \mid Y_{1: k}\right)=p\left(v_{k+1}\right)=N\left(v_{k+1} ; \mathbf{0}, R_{k+1}\right) .
$$

Due to $p\left(y_{k+1} \mid Y_{1: k}\right)$ and $p\left(v_{k+1-r} \mid Y_{1: k}\right)(r=1, \ldots, \min (k+1, s-1))$ being Gaussian, the joint posterior density of $v_{k+1-i}(i=0,1, \ldots, \min (k+1, s-1))$ and $y_{k+1}$ conditioned by $Y_{1: k}$ is also Gaussian, i.e.,

$$
\begin{aligned}
p\left(v_{k+1-i}, y_{k+1} \mid Y_{1: k}\right) & =N\left(\alpha_{k+1-i} ; \hat{\alpha}_{k+1-i \mid k}, \Xi_{k+1-i}^{\alpha}\right) \\
& =\frac{1}{(2 \pi)^{\frac{n_{z}}{2}}\left|P_{k+1-i \mid k+1}^{v v}\right|^{\frac{1}{2}}} \exp \left\{-\frac{\tilde{\alpha}_{k+1-i}^{T} \Xi_{k+1-i} \tilde{\alpha}_{k+1-i}^{T}}{2}\right\} p\left(y_{k+1} \mid Y_{1: k}\right),
\end{aligned}
$$

where $\alpha_{k+1-i}=\left(v_{k+1-i}^{T}, y_{k+1}^{T}\right)^{T}, \hat{\alpha}_{k+1-i \mid k}=E\left(\alpha_{k+1-i \mid Y_{1: k}}\right), \tilde{\alpha}_{k+1-i}=\alpha_{k+1-i}-$

$$
\begin{aligned}
& \hat{\alpha}_{k+1-i}, \Xi_{k+1-i}^{\alpha}=\left[\begin{array}{cc}
P_{k+1-i \mid k}^{v v} & P_{k+1-i, k+1 \mid k}^{v y} \\
\left(P_{k+1-i, k+1 \mid k}^{v y}\right)^{T} & P_{k+1 \mid k}^{y y}
\end{array}\right], \text { and } \Xi_{k+1-i}=\left(\Xi_{k+1-i}^{\alpha}\right)^{-1}- \\
& {\left[\begin{array}{cc}
O & O \\
O & \left(P_{k+1 \mid k}^{y y}\right)^{-1}
\end{array}\right] .}
\end{aligned}
$$

Denote $\tilde{\gamma}_{j, k+1}:=\gamma_{j, k+1}-\sum_{q=0}^{s} \lambda_{q j, k+1} \hat{\mu}_{q, k \mid k}$. Then, $E\left(\tilde{\gamma}_{j, k+1} \mid Y_{1: k}\right)=0$. Furthermore, from $\tilde{y}_{k+1 \mid k}=\sum_{j=0}^{\min (k+1, s)}\left(\gamma_{j, k+1} \tilde{z}_{k+1-j}+\tilde{\gamma}_{j, k+1} \hat{z}_{k+1-j \mid k}\right)$, we have

$$
P_{k+1 \mid k}^{v y}=E\left(v_{k+1} \sum_{j=0}^{\min (k+1, s)} \gamma_{j, k+1} z_{k+1-j} \mid Y_{1: k}\right)=\sum_{q=0}^{s} \lambda_{q 0, k+1} \hat{\mu}_{q, k \mid k} R_{k+1}
$$




$$
\begin{aligned}
& P_{k+1-r, k+1 \mid k}^{v y}=E\left(\tilde{v}_{k+1-r \mid k} \sum_{j=0}^{\min (k+1, s)}\left(\gamma_{j, k+1} \tilde{z}_{k+1-j \mid k}^{T}+\tilde{\gamma}_{j, k+1} \hat{z}_{k+1-j \mid k}^{T}\right) \mid Y_{1: k}\right) \\
= & E\left(\sum_{j=0}^{\min (k+1, s)} E\left(\gamma_{j, k+1} \mid Y_{1: k}\right) E\left(\tilde{v}_{k+1-r \mid k} \tilde{z}_{k+1-j \mid k}^{T}\right) \mid Y_{1: k}\right) \\
= & \sum_{j=0}^{\min (k+1, s)} \sum_{q=0}^{s} \lambda_{q j, k+1} \hat{\mu}_{q, k \mid k}\left(E\left(v_{k+1-r} z_{k+1-j}^{T} \mid Y_{1: k}\right)-\hat{v}_{k+1-r \mid k} \hat{z}_{k+1-j \mid k}^{T}\right)
\end{aligned}
$$

where

$$
\begin{gathered}
E\left(v_{k+1-r} z_{k+1}^{T} \mid Y_{1: k}\right)=\int v_{k+1-r} h_{k+1}^{T}\left(x_{k+1}\right) G_{k+1,0 r \mid k}(\xi) d \xi_{k+1,0 r}, \\
E\left(v_{k+1-r} z_{k+1-l}^{T} \mid Y_{1: k}\right)=E\left(v_{k+1-r} h_{k+1-l}^{T}\left(x_{k+1-l}\right) \mid Y_{1: k}\right)+E\left(v_{k+1-r} v_{k+1-l}^{T} \mid Y_{1: k}\right), \\
=\int v_{k+1-r} h_{k+1-l}^{T}\left(x_{k+1-l}\right) G_{k+1, l r \mid k}(\xi) d \xi_{k+1, l r}+\int v_{k+1-r} v_{k+1-l}^{T} G_{k+1, r l \mid k}(\zeta) d \zeta_{k+1, r l}
\end{gathered}
$$

for $l=1, \cdots, \min (k+1, s)$. Meanwhile, $E\left(v_{k+1-r} v_{k+1} \mid Y_{1: k}\right)=O$.

According to the Bayesian rule, we have

$$
p\left(v_{k+1-i} \mid Y_{1: k+1}\right)=\frac{p\left(v_{k+1-i}, y_{k+1} \mid Y_{1: k}\right)}{p\left(y_{k+1} \mid Y_{1: k}\right)} .
$$

In this way, $p\left(v_{k+1-i} \mid Y_{1: k+1}\right)$ can be updated to be Gaussian, i.e.,

$$
p\left(v_{k+1-i} \mid Y_{1: k+1}\right)=N\left(v_{k+1-i} ; \hat{v}_{k+1-i \mid k+1}, P_{k+1-i \mid k+1}^{v v}\right),
$$

where $\hat{v}_{k+1-i \mid k+1}$ and $P_{k+1-i \mid k+1}^{v v}$ are given by 12 and 13 , respectively. Meanwhile, we can obtain the corresponding gain $K_{k+1-i, k+1}^{v}$ as shown by 14 .

\section{Appendix C. Proof of Theorem 3}

Based on $w_{k}$ being uncorrelated with $Y_{1: k}$, we have

$$
\hat{x}_{k+1 \mid k}=E\left(f_{k}\left(x_{k}\right)+w_{k} \mid Y_{1: k}\right)=E\left(f_{k}\left(x_{k}\right) \mid Y_{1: k}\right)=\int f_{k}\left(x_{k}\right) G_{k \mid k}(x) d x_{k},
$$

and

$$
\begin{aligned}
P_{k+1 \mid k}^{x x} & =E\left(x_{k+1} x_{k+1}^{T} \mid Y_{1: k}\right)-\hat{x}_{k+1 \mid k} \hat{x}_{k+1 \mid k}^{T} \\
& =\int f_{k}\left(x_{k}\right) f_{k}^{T}\left(x_{k}\right) G_{k \mid k}(x) d x_{k}+Q_{k}-\hat{x}_{k+1 \mid k} \hat{x}_{k+1 \mid k}^{T}
\end{aligned}
$$


From Assumptions 1 and 2, we have $p\left(x_{k+1} \mid Y_{1: k}\right)=N\left(x_{k+1} ; \hat{x}_{k+1 \mid k}, P_{k+1 \mid k}^{x x}\right)$ and $p\left(y_{k+1} \mid Y_{k}\right)=N\left(y_{k+1} ; \hat{y}_{k+1 \mid k}, P_{k+1 \mid k}^{y y}\right)$. Meanwhile, $p\left(x_{k+1-r} \mid Y_{1: k}\right)$ is also Gaussian distributed while $\hat{x}_{k+1-r \mid k}$ and $P_{k+1-r \mid k}^{x x}$ have been calculated at the previous $k$ instant for $r=1, \cdots, \min (k+1, s-1)$. Thus, the joint posterior of $x_{k+1-i}$ and $y_{k+1}$ conditioned by $Y_{1: k}$ is also Gaussian, i.e.,

$$
\begin{aligned}
p\left(x_{k+1-i}, y_{k+1} \mid Y_{1: k}\right) & =N\left(\beta_{k+1-i} ; \hat{\beta}_{k+1-i \mid k}, \Im_{k+1-i}^{\beta}\right) \\
& =\frac{1}{(2 \pi)^{\frac{n_{x}}{2}}\left|P_{k+1-i \mid k+1}^{x x}\right|^{\frac{1}{2}}} \exp \left\{-\frac{\tilde{\beta}_{k+1-i}^{T} \Im_{k+1-i} \tilde{\beta}_{k+1-i}}{2}\right\} p\left(y_{k+1} \mid Y_{1: k}\right),
\end{aligned}
$$

where $\beta_{k+1-i}=\left(x_{k+1-i}^{T}, y_{k+1}^{T}\right)^{T}, \hat{\beta}_{k+1-i \mid k}=E\left(\beta_{k+1-i} \mid Y_{1: k}\right), \tilde{\beta}_{k+1-i \mid k}=\beta_{k+1-i}-$ $\hat{\beta}_{k+1-i}, \Im_{k+1-i}^{\beta}=\left[\begin{array}{cc}P_{k+1-i \mid k}^{x x} & P_{k+1-i, k+1 \mid k}^{x y} \\ \left(P_{k+1-i, k+1 \mid k}^{x y}\right)^{T} & P_{k+1 \mid k}^{y y}\end{array}\right]$, and $\Im_{k+1-i}=\left(\Im_{k+1-i}^{\beta}\right)^{-1}-$ $\left[\begin{array}{cc}O & O \\ O & \left(P_{k+1 \mid k}^{y y}\right)^{-1}\end{array}\right]$ for $i=0,1, \cdots, \min (k+1, s-1)$.

Thus, $p\left(x_{k+1-i} \mid Y_{1: k+1}\right)$ can be calculated as Gaussian distributed, i.e.,

$$
p\left(x_{k+1-i} \mid Y_{1: k+1}\right)=N\left(x_{k+1-i} ; \hat{x}_{k+1-i \mid k+1}, P_{k+1-i \mid k+1}^{x x}\right),
$$

where $\hat{x}_{k+1-i \mid k+1}$ and $P_{k+1-i \mid k+1}$ are shown as 25) and 26). Furthermore, we can obtain the corresponding gain $K_{k+1-i, k+1}^{x}$ shown in (27).

Here,

$$
\begin{aligned}
P_{k+1-i, k+1 \mid k}^{x y} & =E\left(\tilde{x}_{k+1-i \mid k} \sum_{j=0}^{\min (k+1, s)}\left(\gamma_{j, k+1} \tilde{z}_{k+1-j \mid k}^{T}+\tilde{\gamma}_{j, k+1} \hat{z}_{k+1-j \mid k}^{T}\right)\right), \\
& =\sum_{j=0}^{\min (k+1, s)} \sum_{q=0}^{s} \lambda_{q j, k+1} \hat{\mu}_{q, k \mid k} P_{k+1-i, k+1-j \mid k}^{x z}
\end{aligned}
$$

where

$$
\begin{gathered}
P_{k+1 \mid k}^{x z}=E\left(x_{k+1} z_{k+1}^{T} \mid Y_{1: k}\right)-\hat{x}_{k+1 \mid k} \hat{z}_{k+1 \mid k}^{T} \\
=\int x_{k+1} h_{k+1}\left(x_{k+1}\right) G_{k+1 \mid k}(x) d x_{k+1}-\hat{x}_{k+1 \mid k} \hat{z}_{k+1 \mid k}^{T}, \\
P_{k+1, k+1-l \mid k}^{x z}=E\left(f_{k}\left(x_{k}\right)\left(h_{k+1-l}\left(x_{k+1-l}\right)+v_{k+1-l}\right)^{T} \mid Y_{1: k}\right)-\hat{x}_{k+1 \mid k} \hat{z}_{k+1-l \mid k}^{T} \\
=\int f_{k}\left(x_{k}\right) h_{k+1-l}^{T}\left(x_{k+1-l}\right) G_{k, 0 l-1 \mid k}(\vartheta) d \vartheta_{k, 0 l-1} \\
+\int f_{k}\left(x_{k}\right) v_{k+1-l}^{T} G_{k, 0 l-1 \mid k}(\xi) d \xi_{k, 0 l-1}-\hat{x}_{k+1 \mid k} \hat{z}_{k+1-l \mid k}^{T}
\end{gathered}
$$




$$
\begin{aligned}
P_{k+1-r, k+1 \mid k}^{x z}= & E\left(x_{k+1-r}\left(h\left(x_{k+1}\right)+v_{k+1}\right)^{T} \mid Y_{1: k}\right)-\hat{x}_{k+1-r \mid k} \hat{z}_{k+1 \mid k}^{T} \\
= & \int x_{k+1-r} h_{k+1}^{T}\left(x_{k+1}\right) G_{k+1, r 0 \mid k}(\vartheta) d \vartheta_{k+1, r 0}-\hat{x}_{k+1-r \mid k} \hat{z}_{k+1 \mid k}^{T} \\
P_{k+1-r, k+1-l \mid k}^{x z}= & E\left(x_{k+1-r}\left(h\left(x_{k+1-l}\right)+v_{k+1-l}\right)^{T} \mid Y_{1: k}\right)-\hat{x}_{k+1-r \mid k} \hat{z}_{k+1-l \mid k}^{T} \\
= & \int x_{k+1-r} h_{k+1-l}^{T}\left(x_{k+1-l}\right) G_{k+1, r l \mid k}(\vartheta) d \vartheta_{k+1, r l} \\
& +\int x_{k+1-r} v_{k+1-l}^{T} G_{k+1, r l \mid k}(\xi) d \xi_{k+1, r l}+\hat{x}_{k+1-r \mid k} \hat{z}_{k+1-l \mid k}^{T}
\end{aligned}
$$

for $l=1, \cdots, \min (k+1, s)$.

\section{Appendix D. Proof of Theorem 4}

From Theorems 2 and 3 , we know that $p\left(x_{k+1-i} \mid Y_{1: t}\right)$ and $p\left(v_{k+1-j} \mid Y_{1: t}\right)(t=$ $k$ or $k+1)$ are all Gaussian distributed for $i, j=0,1, \cdots, \min (k+1, s-1)$. From definitions of $\vartheta_{k+1, i j}, \zeta_{k+1, i j}$ and $\xi_{k+1, i j}$, we can obtain that $p\left(\vartheta_{k+1, i j} \mid Y_{1: t}\right)$, $p\left(\zeta_{k+1, i j} \mid Y_{1: t}\right)$ and $p\left(\xi_{k+1, i j} \mid Y_{1: t}\right)$ will be also updated as Gaussian distributed,

where the first two moments $\hat{\vartheta}_{k+1, i j \mid t}, \hat{\zeta}_{k+1, i j \mid t}, \hat{\xi}_{k+1, i j \mid t}, P_{k+1, i j \mid t}^{\vartheta \vartheta}, P_{k+1, i j \mid t}^{\zeta \zeta}$ and $P_{k+1, i j \mid t}^{\xi \xi}$ can be obtained by (35), (36), (37), 38), 39) and (40), respectively. Meanwhile, from

$K_{k+1-i, k+1}^{\wp} P_{k+1 \mid k}^{y y}\left(K_{k+1-j, k+1}^{\Im}\right)^{T}=K_{k+1-i, k+1}^{\wp}\left(P_{k+1-j, k+1 \mid k}^{\Im y}\right)^{T}=P_{k+1 \mid k}^{\wp y}\left(K_{k+1, k+1-i}^{\Im}\right)^{T}$,

we have

$$
\begin{aligned}
P_{k+1-i, k+1-j \mid k+1}^{\wp \Im} & =E\left(\tilde{\wp}_{k+1-i \mid k}-K_{k+1-i, k+1}^{\wp} \tilde{y}_{k+1 \mid k}\right)\left(\tilde{\Im}_{k+1-j \mid k}-K_{k+1-j, k+1}^{\Im} \tilde{y}_{k+1 \mid k}\right)^{T} \\
& =P_{k+1-i, k+1-j \mid k}^{\wp \Im}-K_{k+1-i, k+1}^{\wp} P_{k+1 \mid k}^{y y}\left(K_{k+1-j, k+1}^{\Im}\right)^{T}
\end{aligned}
$$

where $\wp$ and $\Im$ can be represented as any one of $x$ or $v . P_{k+1 \mid k}^{v v}$ and $P_{k+1 \mid k}^{x x}$ have been obtained using (16) and 29, respectively. Moreover,

$$
\begin{aligned}
P_{k+1, k+1-r \mid k}^{x x} & =E\left(\left(f_{k}\left(x_{k}\right)+w_{k}\right) x_{k+1-r}^{T} \mid Y_{1: k}\right)-\hat{x}_{k+1 \mid k} \hat{x}_{k+1-r \mid k}^{T}, f_{k}\left(x_{k}\right) x_{k+1-r}^{T} G_{k, 0 r-1 \mid k}(\vartheta) d \vartheta_{k, 0 r-1}-\hat{x}_{k+1 \mid k} \hat{x}_{k+1-r \mid k}^{T} \\
& = \\
P_{k+1, k+1-r \mid k}^{x v} & =E\left(\left(f_{k}\left(x_{k}\right)+w_{k}\right) v_{k+1-r}^{T} \mid Y_{1: k}\right)-\hat{x}_{k+1 \mid k} \hat{v}_{k+1-r \mid k}^{T}, \\
& =\int f_{k}\left(x_{k}\right) v_{k+1-r}^{T} G_{k, 0 r-1 \mid k}(\xi) d \xi_{k, 0 r-1}-\hat{x}_{k+1 \mid k} \hat{v}_{k+1-r \mid k}^{T}
\end{aligned},
$$


where $w_{k}$ is uncorrelated with $x_{k+1-r}$ and $v_{k+1-r} . \quad E\left(w_{k} x_{k+1-r}^{T}\right)=O$ and $E\left(w_{k} v_{k+1-r}^{T}\right)=O$. At the same time, due to $v_{k+1}$ being independent of $v_{k+1-l}$ and $x_{k+1-i}$, then, $E\left(v_{k+1-l} v_{k+1}^{T}\right)=O$ and $E\left(x_{k+1-i} v_{k+1}^{T}\right)=O$. Therefore, we can obtain 45 and (46). Here, $l, r=1, \cdots, \min (k+1, s-1)$.

\section{References}

[1] B. Jia, M. Xin, Y. Cheng, Sparse-grid quadrature nonlinear filtering, Automatica 48 (2012) 327-341. doi:10.1016/j.automatica.2011.08.057

[2] G. G. Rigatos, A derivative-free Kalman filtering approach to state estimation-based control of nonlinear systems, IEEE Trans. Industrial Electronics 59 (10) (2012) 3987-3997. doi:10.1109/TIE.2011.2159954.

[3] S. Wang, J. Feng, C. K. Tse, Spherical simplex-radial cubature Kalman filter, IEEE Signal Process. Letters 21 (1) (2014) 43-46. doi:10.1109/LSP. 2013.2290381.

[4] Y. Han, S. Oh, B. Choi, D. Kwak, H. J. Kim, Y. Kim, Fault detection and identification of aircraft control surface using adaptive observer and input bias estimator, IET Control Theory Appl. 6 (10) (2012) 1367-1387. doi:10.1049/iet-cta.2010.0724.

[5] W. Yang, S. Li, N. Li, A switch-mode information fusion filter based on ISRUKF for autonomous navigation of spacecraft, Information Fusion 18 (2014) 33-42. doi:10.1016/j.inffus.2013.04.012

[6] O. Straka, J. Duník, M. Šimandl M, Unscented Kalman filter with advanced

n adaptation of scaling parameter, Automatica 50 (2014) 2657-2664. doi: $375 \quad 10.1016 / \mathrm{j}$.automatica.2014.08.030

[7] B. Jia, M. Xin, Y. Cheng, High-degree cubature Kalman filter, Automatica 49 (2013) 510-518. doi:10.1016/j.automatica.2012.11.014. 
[8] Y. Bar-Shalom, X.R. Li, T. Kirubarajan, Estimation with Applications to Tracking and Navigation: Theory, Algorithms, and Software, Wiley, New York, 2001. doi:10.1002/0471221279.

[9] T. S. Schei, A finite-difference method for linearization in nonlinear estimation algorithms, Automatica 33 (11) 1997 2053-2058. doi:10.1016/ S0005-1098(97)00127-1.

[10] M. Nørgaard, N. K. Poulsen, O. Ravn, New developments in state es385 timation for nonlinear systems, Automatica 36 (2000) 1627-1638. doi: 10.1016/S0005-1098(00)00089-3.

[11] M. Šmandl, J. Duník, Derivative-free estimation methods: new results and performance analysis, Automatica 45 (2009) 1749-1757. doi:10.1016/ j.automatica.2009.03.008

390 [12] G. Terejanu, P. Singla, T. Singh, P. D. Scott, Adaptive Gaussian sum filter for nonlinear Bayesian estimation, IEEE Trans. Automat. Control 56 (9) (2011) 2151-2156. doi:10.1109/TAC.2011.2141550.

[13] S. J. Julier, J. K. Uhlmann, Unscented filtering and nonlinear estimation, 口 Proceedings of the IEEE 92 (3) (2004) 401-422. doi:10.1109/JPROC. 2003. 823141 .

[14] K. Ito, K. Xiong, Gaussian filters for nonlinear filtering problems, IEEE Trans. Automat. Control 45 (5) (2000) 910-927. doi:10.1109/9.855552

[15] I. Arasaratnam, S. Haykin, R. J. Elliott, Discrete-time nonlinear filtering algorithms using Gaussian-Hermite quadrature, Proceedings of the IEEE 95 (5) (2007) 953-977. doi:10.1109/JPROC.2007.894705.

[16] I. Arasaratnam, S. Haykin. Square-root quadrature Kalman filter, IEEE

【 Trans. Signal Process. 56 (6) (2008) 2589-2593. doi:10.1109/TSP. 2007. 914964 . 
[17] I. Arasaratnam, S. Haykin, Cubature Kalman filters, IEEE Trans. Automat. Control 54 (6) (2009) 1254-1269. doi:10.1109/TAC.2009.2019800

[18] J. Ma, S. Sun, Information fusion estimators for systems with multiple sensors of different packet dropout rates, Information Fusion 12 (2011) 213222. doi:10.1016/j.inffus.2010.11.003.

[19] B. Shen, Z. Wang, H. Shu, G. Wei, $H_{\infty}$ filtering for nonlinear discrete-time stochastic systems with randomly varying sensor delays, Automatica, 45 (4) (2009) 1032-1037. doi:10.1016/j.automatica.2008.11.009

[20] Y. Yang, Y. Liang, F. Yang, Y. Qin, Q. Pan. Linear minimum-mean-square error estimation of Markovian jump linear systems with randomly delayed

7 measurements, IET Signal Process. 8 (6) (2014) 658-667. doi:10.1049/ iet-spr.2013.0431.

[21] C. L.Su, C. N. Lu, Interconnected network state estimation using randomly delayed measurements, IEEE Trans. Power Systems 16 (4) (2001) 870-878. doi:10.1109/59.962439

[22] A. Hermoso-Carazo, J. Linares-Pérez, Extended and unscented filtering algorithms using one-step randomly delayed observations, Appl. Math. Comput. 190 (2007) 1375-1393. doi:10.1016/j.amc.2007.02.016

[23] X. Wang, Y. Liang, Q. Puan, C. Zhao, Gaussian filter for nonlinear systems with one-step randomly delayed measurements, Automatica 49 (2013) 976986. doi:10.1016/j.automatica.2013.01.012.

[24] X. Wang, Q. Puan, Y. Liang, F. Yang, Gaussian smoother for nonlinear systems with one-step randomly delayed measurements, IEEE Trans. Automatic. Control 58 (7) (2013) 1828-1835. doi:10.1109/TAC.2013.2237971.

[25] A. Hermoso-Carazo, J. Linares-Pérez, Unscented filtering algorithm using two-step randomly delayed observations in nonlinear systems, Applied 430 \ Mathematical Modelling 33 (2009) 3705-3717. doi:10.1016/j.apm.2008. 12.008 . 
[26] X. He, Z. Wang, Y. D. Ji, D. H. Zhou, Networked-based fault detection for discrete-time state-delay systems: A new measurement model, Int. J. Adapt. Control Signal Process. 22 (2008) 510-528. doi:10.1002/acs.1000

[27] J. Wu, Y. Shi, Consensus in multi-agent systems with random delays govened by a Markov chain, Systems \& Control Letters, 60 (2011) 863-870. doi:10.1016/j.sysconle.2011.07.004.

[28] R. Serfozo, Basics of Applied Stoshastic Processes, Springer, 2009. doi: 10.1007/978-3-540-89332-5.

[29] R. Olfati-Saber, Distributed Kalman filter with embedded consensus filters, Proceedings of the 44th IEEE Conference on Decision and Control, and the European Control Conference, Seville, Spain, 2005, pp. 8179-8184. doi: 10.1109/CDC.2005.1583486.

[30] R. Olfati-Saber, Distributed Kalman filtering for sensor networks, Proceedings of the 46th IEEE conference on decision and control, New Orleans, LA, USA, 2007, pp. 5492-5498. doi:10.1109/CDC.2007.4434303.

[31] R. Olfati-Saber, J. S. Shamma. Consensus filters for sensor networks and distributed sensor fusion, Proceedings of the 44th IEEE conference on decision and control, and the European control conference 2005, Seville, Spain 2005, pp. 6698-6703. doi:10.1109/CDC.2005.1583238.

[32] R. Olfati-Saber, J. A. Fax, R. M. Murray, Consensus and cooperation in networked multi-angent systems, Proceedings of the IEEE 95 (1) (2007) 215-233. doi:10.1109/JPROC.2006.887293.

[33] A. Hermoso-Carazo, J. Linares-Pérez, Nonlinear estimation applying an unscented transformation in systems with correlated uncertain observations,

घ Applied Mathematics and Computation 217 (2011) 7998-8009. doi:10. 1016/j.amc.2011.02.104. 